\title{
Diabetes prevalence and diagnosis in US states: analysis of health
} surveys

\author{
Goodarz Danaei*1,2, Ari B Friedman², Shefali Oza², Christopher JL Murray ${ }^{3}$ \\ and Majid Ezzati ${ }^{1,2}$
}

\author{
Address: ${ }^{1}$ Harvard School of Public Health, Boston, Massachusetts, USA, ${ }^{2}$ Initiative for Global Health, Harvard University, Cambridge, \\ Massachusetts, USA and ${ }^{3}$ Institute for Health Metrics and Evaluation, University of Washington, Seattle, Washington, USA \\ Email: Goodarz Danaei* - gdanaei@hsph.harvard.edu; Ari B Friedman - abfriedman@gmail.com; Shefali Oza - shefalita@gmail.com; \\ Christopher JL Murray - cjlm@u.washington.edu; Majid Ezzati - majid_ezzati@harvard.edu \\ * Corresponding author
}

Published: 25 September 2009

Population Health Metrics 2009, 7:16 doi:10.1186/1478-7954-7-16
Received: 5 March 2009

Accepted: 25 September 2009

This article is available from: http://www.pophealthmetrics.com/content/7/1/16

(C) 2009 Danaei et al; licensee BioMed Central Ltd.

This is an Open Access article distributed under the terms of the Creative Commons Attribution License (http://creativecommons.org/licenses/by/2.0), which permits unrestricted use, distribution, and reproduction in any medium, provided the original work is properly cited.

\begin{abstract}
Background: Current US surveillance data provide estimates of diabetes using laboratory tests at the national level as well as self-reported data at the state level. Self-reported diabetes prevalence may be biased because respondents may not be aware of their risk status. Our objective was to estimate the prevalence of diagnosed and undiagnosed diabetes by state.

Methods: We estimated undiagnosed diabetes prevalence as a function of a set of health system and sociodemographic variables using a logistic regression in the National Health and Nutrition Examination Survey (2003-2006). We applied this relationship to identical variables from the Behavioral Risk Factor Surveillance System (2003-2007) to estimate state-level prevalence of undiagnosed diabetes by age group and sex. We assumed that those who report being diagnosed with diabetes in both surveys are truly diabetic.

Results: The prevalence of diabetes in the U.S. was 13.7\% among men and II.7\% among women $\geq 30$ years. Age-standardized diabetes prevalence was highest in Mississippi, West Virginia, Louisiana, Texas, South Carolina, Alabama, and Georgia (I5.8 to 16.6\% for men and I2.4 to 14.8\% for women). Vermont, Minnesota, Montana, and Colorado had the lowest prevalence (II.0 to 12.2\% for men and 7.3 to $8.4 \%$ for women). Men in all states had higher diabetes prevalence than women. The absolute prevalence of undiagnosed diabetes, as a percent of total population, was highest in New Mexico, Texas, Florida, and California (3.5 to 3.7 percentage points) and lowest in Montana, Oklahoma, Oregon, Alaska, Vermont, Utah, Washington, and Hawaii (2.I to 3 percentage points). Among those with no established diabetes diagnosis, being obese, being Hispanic, not having insurance and being $\geq 60$ years old were significantly associated with a higher risk of having undiagnosed diabetes.
\end{abstract}

Conclusion: Diabetes prevalence is highest in the Southern and Appalachian states and lowest in the Midwest and the Northeast. Better diabetes diagnosis is needed in a number of states. 


\section{Background}

Diabetes Mellitus is the sixth leading cause of death in the United States (U.S.), accounting for approximately 70,000 annual deaths. Age-standardized adult diabetes death rates across U.S. states ranged from approximately 2 per 10,000 people in Arizona and Florida to 4.5 to 5 in West Virginia and the District of Columbia (D.C.) [1]. There may be two reasons for this large variation: First, there may be variation in diabetes prevalence across states due to differences in risk factors for diabetes. For example, the prevalence of obesity in a number of Southern states is almost $60 \%$ higher than Colorado, where obesity is lowest [2]. Second, there may be differences across states in diagnosis and treatment of diabetes or of cardiovascular risks among diabetics. Reliable information on diagnosed and undiagnosed diabetes prevalence at the state level is important because states are important administrative units for funding and implementing programs that influence diagnosis and treatment.

Currently, the only source of information on diabetes prevalence at the state level is the Behavioral Risk Factor Surveillance System (BRFSS), a state-representative telephone survey. However, the BRFSS data are based on selfreports and do not provide estimates of undiagnosed diabetes. The National Health and Nutrition Examination Survey (NHANES) uses laboratory measurements and provides estimates of diagnosed and undiagnosed diabetes, but is representative only at the national level. In this study, we combined data from NHANES and BRFSS to estimate diabetes prevalence and diagnosis at the state level. Our results provide information for state diabetes prevention and control programs, and our methods can be used for regular low-cost monitoring of diabetes at the state level.

\section{Methods \\ Data Sources}

NHANES uses a complex multistage stratified clustered probability design to measure health and nutrition characteristics of a nationally representative sample of the civilian non-institutionalized population aged two months and older. NHANES includes an in-person interview and a subsequent physical examination and measurement component in a mobile examination clinic (MEC) or at home for those unable to visit the MEC. We used NHANES data from 2003 to 2006. The response rates for the household interviews were $80 \%$ for 2003 2004 and $79 \%$ for 2005-2006. The corresponding response rates for the medical examination after the household interview were 95 to $96 \%$.

Each interviewed participant was randomly assigned to either a morning or afternoon/evening MEC session. Subjects $\geq 20$ years old assigned to the morning session were asked to fast for 8 to 24 hours, with the exception of those on insulin or those who were excluded for other safety reasons. The NHANES MEC and fasting sample weights account for exclusion, non-response, and inappropriate fasting time. Additional information on NHANES design and methods, including on diabetes measurement, is available elsewhere $[3,4]$ and online http://www.cdc.gov/ nchs/nhanes.htm.

The BRFSS is an annual cross-sectional telephone health survey. Currently, the survey is conducted in all 50 states and the District of Columbia using random-digit dialing to obtain a state-representative sample of the civilian, non-institutionalized population aged 18 and over. In 2003, the response rate among eligible subjects who answered the phone was $77 \%$. Additional information on the design is available elsewhere [5,6] and online http:// www.cdc.gov/brfss.

We included adults aged 30 and older in NHANES and BRFSS who had answered the self-reported diabetes question, which asked if they had ever been told by a health professional that they had diabetes. The response rate for this question was more than $99.8 \%$ in both surveys. We did not include younger participants because diabetes prevalence is relatively low in these ages.

\section{Statistical Analysis}

Consistent with previous analyses [4], we defined total diabetes as either having answered yes to the diabetes diagnosis question: "Other than during pregnancy, have you ever been told by a doctor or health professional that you have diabetes or sugar diabetes?" or having a fasting plasma glucose (FPG) level of $\geq 126 \mathrm{mg} / \mathrm{dL}$. We used FPG because it is used to define diabetes by the American Diabetes Association [7].

We used data from NHANES, which is representative at the national but not at the state level, to characterize the relationship between undiagnosed diabetes status (defined as FPG $\geq 126 \mathrm{mg} / \mathrm{dL}$ ) and a set of health system, sociodemographic, and risk factor variables listed in Table 1 using a logistic regression. These variables were selected a priori based on their potential association with diabetes prevalence. We excluded education from the primary list of predictors as including it did not improve the fit of the model. In addition, $50.2 \%$ of observations in NHANES were missing either smoking or insurance status or both. We used a missing indicator to include these observations in the regression model. The regression incorporated appropriate sampling weights.

We estimated the individual-level probability of having diabetes in BRFSS 2003-2007 in two steps: First, participants who had answered "yes" to the diabetes diagnosis question were, by definition, assigned a probability of 1.0 for having diabetes. Second, the probability of having 
Table I: Description of the outcome and explanatory variables from NHANES and BRFSS and the corresponding odds ratios (OR) and $95 \%$ confidence intervals $(95 \% \mathrm{Cl})$.

\begin{tabular}{|c|c|c|c|c|}
\hline Variable & Reason for inclusion in analysis & Possible values & OR & $(95 \% \mathrm{Cl})$ \\
\hline \multicolumn{5}{|c|}{ Outcome (dependent) variables for the regression } \\
\hline $\begin{array}{l}\text { Undiagnosed diabetes } \\
\text { (available in NHANES; predicted in } \\
\text { BRFSS) }\end{array}$ & $\begin{array}{l}\text { Outcome variable to estimate undiagnosed } \\
\text { diabetes }\end{array}$ & $\begin{array}{l}0(\text { FPG }<126 \mathrm{mg} / \mathrm{dL}) \\
\text { I (FPG } \geq 126 \mathrm{mg} / \mathrm{dL})\end{array}$ & - & - \\
\hline \multicolumn{5}{|c|}{ Explanatory (independent) variables } \\
\hline \multirow[t]{2}{*}{ Sex } & $\begin{array}{l}\text { Predictor of diabetes, possibly because of } \\
\text { differences in lifestyle and health care } \\
\text { determinants }\end{array}$ & Male & 1.0 & - \\
\hline & & Female & 0.47 & $0.29,0.77$ \\
\hline \multirow[t]{5}{*}{ Age (years) } & Predictor of diabetes & $30-39$ & 1.0 & - \\
\hline & & $40-49$ & 1.07 & $0.41,2.81$ \\
\hline & & $50-59$ & 3.25 & I.4I, 7.50 \\
\hline & & $60-69$ & 7.49 & $3.55,15.82$ \\
\hline & & $\geq 70$ & 7.02 & $3.23,15.26$ \\
\hline \multirow[t]{4}{*}{ Race * } & Predictor of diabetes and health care access & Non-Hispanic white & 1.0 & - \\
\hline & & Non-Hispanic black & 1.11 & $0.63,1.96$ \\
\hline & & Hispanic & 2.03 & $1.07,3.83$ \\
\hline & & Other & 0.26 & $0.03,1.91$ \\
\hline \multirow{2}{*}{$\begin{array}{l}\text { Doctor visit (have you seen a doctor in } \\
\text { the past year?) } \dagger\end{array}$} & Indicator for diabetes knowledge and control & No & 0.49 & $0.23,1.05$ \\
\hline & & Yes & 1.0 & - \\
\hline \multirow{3}{*}{$\begin{array}{l}\text { Insurance status } \\
\text { (do you currently have health insurance?) }\end{array}$} & Indicator for diabetes knowledge and control & No & 1.58 & $0.83,3.02$ \\
\hline & & Yes & 1.0 & \\
\hline & & Missing & 0.76 & $0.07,8.42$ \\
\hline \multirow[t]{3}{*}{ BMI $\left(\mathrm{kg} / \mathrm{m}^{2}\right) \ddagger$} & $\begin{array}{l}\text { Determinant of diabetes and indicator for } \\
\text { selected lifestyle factors such as diet and } \\
\text { physical activity }\end{array}$ & $<25$ & 1.0 & - \\
\hline & & $25-29$ & 1.85 & $0.93,3.67$ \\
\hline & & $\geq 30$ & 4.29 & $2.25,8.17$ \\
\hline \multirow[t]{3}{*}{ Smoking (do you now smoke cigarettes?) } & Indicator for lifestyle factors & Yes ("everyday" or "some days") & 1.0 & - \\
\hline & & No ("not at all") & 1.27 & $0.65,2.49$ \\
\hline & & Missing & 0.86 & $0.51,1.45$ \\
\hline
\end{tabular}

* We combined Mexican Americans and other Hispanics in NHANES to match the race categories in BRFSS.

† This variable was defined as a composite of multiple questions about physician contact for specific conditions in the BRFSS.

$\ddagger \mathrm{BMI}$ was corrected for bias in self-reported height and weight in telephone interviews, using methods described elsewhere [2]. 
undiagnosed diabetes (i.e., FPG $\geq 126 \mathrm{mg} / \mathrm{dL}$ ) for those who answered "no" to this question was estimated using the coefficients of the logistic regression fit on the NHANES dataset. Estimates of diabetes prevalence and diabetes diagnosis by age, sex, and state were obtained from the BRFSS using appropriate sample weights. The difference between total diabetes and self-reported diabetes is undiagnosed diabetes. In separate analyses, we used linear regressions to model the relationship between FPG as a continuous variable and self-reported diabetes diagnosis, medication use, and the health system, sociodemographic, and risk factor variables in Table 1 (results for continuous FPG analysis are available from authors by request). We used STATA version 10 for all analyses (StataCorp Texas). We present the results in two age groups: 3059 and $\geq 60$ years.

\section{Results}

The national prevalence of diabetes among US adults $\geq 30$ years was $13.7 \%$ (95\% Confidence Interval $12.0 \%$, $15.4 \%$ ) for men and $11.7 \%$ (CI95 10.4\%, 13.0\%) for women in the pooled 2003-2006 NHANES. Nationally, approximately 32\% of all diabetes cases in 2003-2006 were undiagnosed, a percentage that has changed little since 1999-2002 [4].

\section{Regression results}

Among those who answered "no" to having been diagnosed with diabetes, being male and being older was associated with a higher probability of having diabetes (Table $1)$. The effect of age on diabetes risk was largest in those 60 to 69 years old and declined slightly in those $\geq 70$ years old, consistent with the available evidence on the age association of blood glucose [8]. Overweight and obesity were associated with higher prevalence of undiagnosed diabetes, with obese participants (body mass index, BMI $\geq 30 \mathrm{~kg} / \mathrm{m}^{2}$ ) having 4.29 times (95\% CI 2.25, 8.17) the odds of having undiagnosed diabetes compared to normal weight. After controlling for all other factors, Hispanics had twice $(95 \%$ CI $1.07,3.83)$ the odds of having undiagnosed diabetes compared to whites, and the uninsured had 1.58 (95\% CI $0.83,3.02)$ times the odds compared to insured subjects.

We evaluated the performance of the prediction model using both internal and external validations. For internal validation, we applied the regression coefficients to NHANES 2003-2006 observations (i.e., the same data used in estimating the regression model) to predict diabetes prevalence. The differences between the predicted and actual diabetes prevalence for different age, sex, and race groups were on average 0.5 percentage points and at most 8.4 percentage points. The Pearson correlation coefficient for the observed and predicted diabetes prevalence for different age, sex, and race groups was 0.98 . For external val- idation, we applied the coefficients of regressions estimated using the 2003- 2006 rounds to the same variables in pooled data from two previous rounds of NHANES (1999-2000 and 2001-2002). The observed-predicted differences for individual age, sex, and race groups were at the extreme slightly worse than those in the internal validation; specifically, the 60- to 69-year-old males from "other race" had a 20 percentage point discrepancy. This may, however, be because the composition of this race changed between the two surveys. The Pearson correlation coefficient for the observed and predicted diabetes prevalence for different age, sex, and race groups was 0.93 . On average, the predicted prevalence was 0.1 percentage points higher than the actual prevalence (versus 0.5 lower percentage points in the internal validation).

\section{National-level prevalence of diabetes and undiagnosed diabetes}

The predicted national prevalence of diabetes in 20032007 was $14.4 \%(14.3 \%, 14.5 \%)$ for men and $11.4 \%$ $(11.3 \%, 11.5 \%)$ in women. The only sociodemographic group whose predicted and measured prevalences were significantly different was the uninsured, who had an actual prevalence of $9.2 \%(7.4 \%, 11.0 \%)$ but a predicted prevalence of $11.9 \%(11.6 \%, 12.2 \%)$.

\section{State-level prevalence of diabetes and undiagnosed diabetes}

In 2003-2007, the lowest prevalence of diabetes was in the Midwest and the Northeast, including Vermont, Minnesota, Montana, and Colorado, with age-standardized prevalence ranging from $11.0 \%$ to $12.2 \%$ for men and $7.3 \%$ to $8.4 \%$ for women (Figure 1 and Table 2 ). Diabetes prevalence was highest in the primarily Southern and Appalachian states, including Mississippi, West Virginia, Louisiana, Texas, South Carolina, Alabama, and Georgia, where age-standardized diabetes prevalence was $15.8 \%$ to $16.6 \%$ for men and $12.4 \%$ to $14.8 \%$ for women, i.e., approximately $30 \%$ to $51 \%$ higher for men and $48 \%$ to $103 \%$ higher for women than the states with lowest prevalence. The same geographic pattern was observed when younger (30-59 years) and older ( $\geq 60$ years) age groups were considered separately. The Spearman rank correlation coefficient of state diabetes prevalence and mean BMI was 0.53 for men and 0.76 for women [2].

Age-standardized diabetes prevalence was higher in men than women in all states, with the largest differences in Minnesota, Colorado, Utah, and Maine, where prevalence in men was $32 \%$ to $38 \%$ higher than among women. The smallest male-female differences were in the District of Columbia, Mississippi, West Virginia, and Louisiana, ranging from $6 \%$ to $18 \%$ (Figures 1 and 2 ). Men also had higher prevalence of diabetes than women in almost all states and age groups, except in the youngest ages (30 to 
Table 2: Estimated prevalence (sampling standard error)* of total diabetes by state, age, sex, race and insurance status (Figures show actual prevalence; age-standardized figures available from authors).

\begin{tabular}{|c|c|c|c|c|c|c|c|c|c|c|}
\hline \multirow[t]{2}{*}{ State } & \multicolumn{2}{|c|}{ Age group } & \multicolumn{2}{|c|}{ Sex } & \multicolumn{4}{|c|}{ Race } & \multicolumn{2}{|c|}{ Insurance status } \\
\hline & $30-59 y$ & $\geq 60 y$ & Men & Women & White & Black & Hispanic & Other races & Insured & Uninsured \\
\hline National NHANES & $\begin{array}{c}8.4 \% \\
(.6)\end{array}$ & $\begin{array}{c}23.6 \% \\
(.1)\end{array}$ & $\begin{array}{c}13.7 \% \\
(.8)\end{array}$ & $\begin{array}{c}11.7 \% \\
(.7)\end{array}$ & $\begin{array}{c}11.4 \% \\
(.7)\end{array}$ & $\begin{array}{c}18.3 \% \\
(1.0)\end{array}$ & $\begin{array}{c}16.7 \% \\
(1.6)\end{array}$ & $\begin{array}{l}11.1 \% \\
(1.5)\end{array}$ & $\begin{array}{c}13.3 \% \\
(.7)\end{array}$ & $\begin{array}{l}9.2 \% \\
(.9)\end{array}$ \\
\hline National BRFSS Prediction & $\begin{array}{l}8.4 \% \\
(.05)\end{array}$ & $\begin{array}{c}23.8 \% \\
(.1)\end{array}$ & $\begin{array}{c}14.4 \% \\
(.07)\end{array}$ & $\begin{array}{c}11.4 \% \\
(.05)\end{array}$ & $\begin{array}{c}11.9 \% \\
(.04)\end{array}$ & $\begin{array}{c}17.3 \% \\
(.18)\end{array}$ & $\begin{array}{c}16.3 \% \\
(.26)\end{array}$ & $\begin{array}{l}11.3 \% \\
(.29)\end{array}$ & $\begin{array}{c}13.0 \% \\
(.05)\end{array}$ & $\begin{array}{l}11.9 \% \\
(.15)\end{array}$ \\
\hline Alabama & $\begin{array}{c}10.1 \% \\
(.3)\end{array}$ & $\begin{array}{l}25 \% \\
(.54)\end{array}$ & $\begin{array}{c}16.1 \% \\
(.45)\end{array}$ & $\begin{array}{c}13.5 \% \\
(.33)\end{array}$ & $\begin{array}{c}13.6 \% \\
(.3)\end{array}$ & $\begin{array}{l}19 \% \\
(.69)\end{array}$ & $\begin{array}{l}14.5 \% \\
(1.91)\end{array}$ & $\begin{array}{l}11.7 \% \\
(1.81)\end{array}$ & $\begin{array}{l}15 \% \\
(.3)\end{array}$ & $\begin{array}{c}12.4 \% \\
(.71)\end{array}$ \\
\hline Alaska & $\begin{array}{c}6 \% \\
(.31)\end{array}$ & $\begin{array}{l}21.8 \% \\
(1.09)\end{array}$ & $\begin{array}{c}9.9 \% \\
(.5)\end{array}$ & $\begin{array}{l}8.2 \% \\
(.47)\end{array}$ & $\begin{array}{l}9.2 \% \\
(.38)\end{array}$ & $\begin{array}{c}8.9 \% \\
(1.17)\end{array}$ & $\begin{array}{l}11.7 \% \\
(2.25)\end{array}$ & $\begin{array}{c}8.3 \% \\
(1.01)\end{array}$ & $\begin{array}{l}9.3 \% \\
(.38)\end{array}$ & $\begin{array}{l}7.8 \% \\
(.77)\end{array}$ \\
\hline Arizona & $\begin{array}{l}8.4 \% \\
(.41)\end{array}$ & $\begin{array}{c}22.1 \% \\
(.65)\end{array}$ & $\begin{array}{c}14.5 \% \\
(.58)\end{array}$ & $\begin{array}{c}10.7 \% \\
(.42)\end{array}$ & $\begin{array}{c}11.7 \% \\
(.38)\end{array}$ & $\begin{array}{c}15 \% \\
(1.66)\end{array}$ & $\begin{array}{l}17.8 \% \\
(1.28)\end{array}$ & $\begin{array}{l}11.3 \% \\
(1.32)\end{array}$ & $\begin{array}{c}12.9 \% \\
(.39)\end{array}$ & $\begin{array}{l}11.1 \% \\
(.94)\end{array}$ \\
\hline Arkansas & $\begin{array}{l}9.1 \% \\
(.24)\end{array}$ & $\begin{array}{c}21.4 \% \\
(.42)\end{array}$ & $\begin{array}{c}14.5 \% \\
(.35)\end{array}$ & $\begin{array}{c}11.7 \% \\
(.27)\end{array}$ & $\begin{array}{c}12.6 \% \\
(.23)\end{array}$ & $\begin{array}{l}17 \% \\
(.8)\end{array}$ & $\begin{array}{l}13.6 \% \\
(1.66)\end{array}$ & $\begin{array}{l}12.7 \% \\
(1.33)\end{array}$ & $\begin{array}{c}13.5 \% \\
(.24)\end{array}$ & $\begin{array}{c}10.5 \% \\
(.51)\end{array}$ \\
\hline California & $\begin{array}{l}8.3 \% \\
(.25)\end{array}$ & $\begin{array}{l}25 \% \\
(.59)\end{array}$ & $\begin{array}{c}14.4 \% \\
(.4)\end{array}$ & $\begin{array}{l}11 \% \\
(.3)\end{array}$ & $\begin{array}{c}10.7 \% \\
(.25)\end{array}$ & $\begin{array}{c}15.4 \% \\
(.86)\end{array}$ & $\begin{array}{c}16.2 \% \\
(.61)\end{array}$ & $\begin{array}{c}10.5 \% \\
(.94)\end{array}$ & $\begin{array}{c}12.9 \% \\
(.27)\end{array}$ & $\begin{array}{c}11.2 \% \\
(.72)\end{array}$ \\
\hline Colorado & $\begin{array}{l}5.7 \% \\
\text { (.17) }\end{array}$ & $\begin{array}{c}18.9 \% \\
(.41)\end{array}$ & $\begin{array}{c}10.6 \% \\
(.26)\end{array}$ & $\begin{array}{l}7.3 \% \\
(.2)\end{array}$ & $\begin{array}{c}8 \% \\
(.17)\end{array}$ & $\begin{array}{c}11.4 \% \\
(.86)\end{array}$ & $\begin{array}{l}15 \% \\
(.67)\end{array}$ & $\begin{array}{c}9.2 \% \\
(1.13)\end{array}$ & $\begin{array}{l}9.1 \% \\
(.18)\end{array}$ & $\begin{array}{l}7.7 \% \\
(.45)\end{array}$ \\
\hline Connecticut & $\begin{array}{l}6.5 \% \\
(.19)\end{array}$ & $\begin{array}{c}20.6 \% \\
(.4)\end{array}$ & $\begin{array}{c}12.4 \% \\
(.29)\end{array}$ & $\begin{array}{l}9.3 \% \\
(.23)\end{array}$ & $\begin{array}{c}10.3 \% \\
(.19)\end{array}$ & $\begin{array}{c}16.7 \% \\
(1)\end{array}$ & $\begin{array}{c}14.6 \% \\
(.97)\end{array}$ & $\begin{array}{c}8.9 \% \\
(1.22)\end{array}$ & $\begin{array}{c}10.8 \% \\
(.19)\end{array}$ & $\begin{array}{c}10.8 \% \\
(.68)\end{array}$ \\
\hline Delaware & $\begin{array}{l}8.3 \% \\
(.31)\end{array}$ & $\begin{array}{c}23.1 \% \\
(.56)\end{array}$ & $\begin{array}{l}15 \% \\
(.45)\end{array}$ & $\begin{array}{c}10.9 \% \\
(.34)\end{array}$ & $\begin{array}{c}12.4 \% \\
(.3)\end{array}$ & $\begin{array}{c}16.4 \% \\
(.92)\end{array}$ & $\begin{array}{c}12.5 \% \\
(2)\end{array}$ & $\begin{array}{c}9.3 \% \\
(1.35)\end{array}$ & $\begin{array}{l}13 \% \\
(.29)\end{array}$ & $\begin{array}{c}10.7 \% \\
(.95)\end{array}$ \\
\hline District of Columbia & $\begin{array}{l}8.1 \% \\
(.31)\end{array}$ & $\begin{array}{c}26.3 \% \\
(.78)\end{array}$ & $\begin{array}{c}12.9 \% \\
(.48)\end{array}$ & $\begin{array}{c}13.3 \% \\
(.44)\end{array}$ & $\begin{array}{l}5.4 \% \\
(.24)\end{array}$ & $\begin{array}{c}18.1 \% \\
(.52)\end{array}$ & $\begin{array}{r}9.2 \% \\
(1.24)\end{array}$ & $\begin{array}{c}9.7 \% \\
(1.72)\end{array}$ & $\begin{array}{c}13.1 \% \\
(.34)\end{array}$ & $\begin{array}{l}12.8 \% \\
(1.05)\end{array}$ \\
\hline Florida & $\begin{array}{c}9 \% \\
(.25)\end{array}$ & $\begin{array}{c}23.1 \% \\
(.39)\end{array}$ & $\begin{array}{c}16.2 \% \\
(.36)\end{array}$ & $\begin{array}{l}12 \% \\
(.26)\end{array}$ & $\begin{array}{l}13 \% \\
(.23)\end{array}$ & $\begin{array}{l}18 \% \\
(.83)\end{array}$ & $\begin{array}{c}15.9 \% \\
(.7)\end{array}$ & $\begin{array}{l}11.7 \% \\
(1.54)\end{array}$ & $\begin{array}{c}14.3 \% \\
(.24)\end{array}$ & $\begin{array}{c}12.3 \% \\
(.61)\end{array}$ \\
\hline Georgia & $\begin{array}{l}9.2 \% \\
(.25)\end{array}$ & $\begin{array}{c}26.5 \% \\
(.5)\end{array}$ & $\begin{array}{c}14.6 \% \\
(.37)\end{array}$ & $\begin{array}{c}12.2 \% \\
(.28)\end{array}$ & $\begin{array}{c}12.2 \% \\
(.24)\end{array}$ & $\begin{array}{c}16.9 \% \\
(.57)\end{array}$ & $\begin{array}{l}12.2 \% \\
(1.39)\end{array}$ & $\begin{array}{r}9.9 \% \\
(1.25)\end{array}$ & $\begin{array}{c}13.5 \% \\
(.25)\end{array}$ & $\begin{array}{c}12.2 \% \\
(.62)\end{array}$ \\
\hline Hawaii & $\begin{array}{c}7.6 \% \\
(.3)\end{array}$ & $\begin{array}{c}20.8 \% \\
(.59)\end{array}$ & $\begin{array}{c}12.9 \% \\
(.44)\end{array}$ & $\begin{array}{c}10.6 \% \\
(.36)\end{array}$ & $\begin{array}{l}8.7 \% \\
(.34)\end{array}$ & $\begin{array}{l}14 \% \\
(.7)\end{array}$ & $\begin{array}{l}14.2 \% \\
(1.28)\end{array}$ & $\begin{array}{c}12.4 \% \\
(.46)\end{array}$ & $\begin{array}{c}11.9 \% \\
(.29)\end{array}$ & $\begin{array}{l}9.3 \% \\
(.89)\end{array}$ \\
\hline Idaho & $\begin{array}{l}7.7 \% \\
(.24)\end{array}$ & $\begin{array}{c}21.7 \% \\
(.45)\end{array}$ & $\begin{array}{c}12.8 \% \\
(.35)\end{array}$ & $\begin{array}{c}10.6 \% \\
(.27)\end{array}$ & $\begin{array}{c}11.4 \% \\
(.23)\end{array}$ & $\begin{array}{l}16.1 \% \\
(1.97)\end{array}$ & $\begin{array}{l}16.4 \% \\
(1.45)\end{array}$ & $\begin{array}{l}13.4 \% \\
(1.45)\end{array}$ & $\begin{array}{l}12 \% \\
(.24)\end{array}$ & $\begin{array}{l}10 \% \\
(.56)\end{array}$ \\
\hline Illinois & $\begin{array}{l}8.6 \% \\
(.27)\end{array}$ & $\begin{array}{c}23.8 \% \\
(.49)\end{array}$ & $\begin{array}{c}14.5 \% \\
(.4)\end{array}$ & $\begin{array}{c}11.2 \% \\
(.28)\end{array}$ & $\begin{array}{l}11.2 \% \\
(.22)\end{array}$ & $\begin{array}{c}18.6 \% \\
(.88)\end{array}$ & $\begin{array}{l}17.6 \% \\
(1.33)\end{array}$ & $\begin{array}{r}9.8 \% \\
(1.49)\end{array}$ & $\begin{array}{c}12.5 \% \\
(.24)\end{array}$ & $\begin{array}{l}15.9 \% \\
(1.06)\end{array}$ \\
\hline Indiana & $\begin{array}{l}8.4 \% \\
(.22)\end{array}$ & $\begin{array}{c}24.8 \% \\
(.45)\end{array}$ & $\begin{array}{c}14.7 \% \\
(.33)\end{array}$ & $\begin{array}{c}11.9 \% \\
(.26)\end{array}$ & $\begin{array}{c}12.9 \% \\
(.22)\end{array}$ & $\begin{array}{c}18 \% \\
(1.01)\end{array}$ & $\begin{array}{c}15.7 \% \\
(1.7)\end{array}$ & $\begin{array}{l}10.9 \% \\
(1.35)\end{array}$ & $\begin{array}{c}13.3 \% \\
(.22)\end{array}$ & $\begin{array}{c}12.3 \% \\
(.63)\end{array}$ \\
\hline
\end{tabular}


Table 2: Estimated prevalence (sampling standard error)* of total diabetes by state, age, sex, race and insurance status (Figures show actual prevalence; age-standardized figures available from authors). (Continued)

\begin{tabular}{|c|c|c|c|c|c|c|c|c|c|c|}
\hline lowa & $\begin{array}{c}6.9 \% \\
(.2)\end{array}$ & $\begin{array}{c}22.1 \% \\
(.4 I)\end{array}$ & $\begin{array}{c}13.4 \% \\
(.31)\end{array}$ & $\begin{array}{l}10.3 \% \\
(.25)\end{array}$ & $\begin{array}{c}11.8 \% \\
(.2)\end{array}$ & $\begin{array}{l}14.7 \% \\
(1.69)\end{array}$ & $\begin{array}{l}15.7 \% \\
(1.85)\end{array}$ & $\begin{array}{c}4.6 \% \\
(1.15)\end{array}$ & $\begin{array}{l}11.9 \% \\
(.21)\end{array}$ & $\begin{array}{c}10.5 \% \\
(.69)\end{array}$ \\
\hline Kansas & $\begin{array}{l}7.3 \% \\
(.17)\end{array}$ & $\begin{array}{c}21.7 \% \\
(.35)\end{array}$ & $\begin{array}{l}13 \% \\
(.26)\end{array}$ & $\begin{array}{c}10.4 \% \\
(.21)\end{array}$ & $\begin{array}{l}11.3 \% \\
(.17)\end{array}$ & $\begin{array}{c}17.1 \% \\
(.95)\end{array}$ & $\begin{array}{l}14.3 \% \\
(1.03)\end{array}$ & $\begin{array}{l}10.6 \% \\
(1.17)\end{array}$ & $\begin{array}{l}11.8 \% \\
(.17)\end{array}$ & $\begin{array}{c}10.7 \% \\
(.55)\end{array}$ \\
\hline Kentucky & $\begin{array}{l}9.8 \% \\
(.27)\end{array}$ & $\begin{array}{c}24.7 \% \\
(.47)\end{array}$ & $\begin{array}{c}15.7 \% \\
(.4)\end{array}$ & $\begin{array}{c}12.5 \% \\
(.28)\end{array}$ & $\begin{array}{c}13.7 \% \\
(.24)\end{array}$ & $\begin{array}{l}20.8 \% \\
(1.55)\end{array}$ & $\begin{array}{l}15.1 \% \\
(1.96)\end{array}$ & $\begin{array}{l}11.8 \% \\
(1.83)\end{array}$ & $\begin{array}{c}14.3 \% \\
(.26)\end{array}$ & $\begin{array}{c}12.2 \% \\
(.61)\end{array}$ \\
\hline Louisiana & $\begin{array}{l}10 \% \\
(.26)\end{array}$ & $\begin{array}{c}26.6 \% \\
(.5)\end{array}$ & $\begin{array}{c}15.7 \% \\
(.38)\end{array}$ & $\begin{array}{c}13.6 \% \\
(.29)\end{array}$ & $\begin{array}{l}13 \% \\
(.26)\end{array}$ & $\begin{array}{c}18.8 \% \\
(.56)\end{array}$ & $\begin{array}{l}16.3 \% \\
(1.61)\end{array}$ & $\begin{array}{l}13.1 \% \\
(1.31)\end{array}$ & $\begin{array}{c}14.5 \% \\
(.26)\end{array}$ & $\begin{array}{c}15.1 \% \\
(.59)\end{array}$ \\
\hline Maine & $\begin{array}{l}7.6 \% \\
(.26)\end{array}$ & $\begin{array}{c}22.2 \% \\
(.52)\end{array}$ & $\begin{array}{c}14.1 \% \\
(.4)\end{array}$ & $\begin{array}{c}10.3 \% \\
(.29)\end{array}$ & $\begin{array}{l}12.1 \% \\
(.25)\end{array}$ & $\begin{array}{l}16.6 \% \\
(3.06)\end{array}$ & $\begin{array}{l}11.2 \% \\
(1.78)\end{array}$ & $\begin{array}{l}11.4 \% \\
(1.8)\end{array}$ & $\begin{array}{c}12.4 \% \\
(.26)\end{array}$ & $\begin{array}{l}9.2 \% \\
(.65)\end{array}$ \\
\hline Maryland & $\begin{array}{l}7.7 \% \\
(.22)\end{array}$ & $\begin{array}{c}24.7 \% \\
(.51)\end{array}$ & $\begin{array}{c}13.5 \% \\
(.35)\end{array}$ & $\begin{array}{l}11 \% \\
(.28)\end{array}$ & $\begin{array}{l}11.2 \% \\
(.21)\end{array}$ & $\begin{array}{c}15.4 \% \\
(.57)\end{array}$ & $\begin{array}{c}12 \% \\
(1.71)\end{array}$ & $\begin{array}{r}8.8 \% \\
(1.12)\end{array}$ & $\begin{array}{c}12.3 \% \\
(.23)\end{array}$ & $\begin{array}{c}10.6 \% \\
(.77)\end{array}$ \\
\hline Massachusetts & $\begin{array}{l}6.4 \% \\
(.16)\end{array}$ & $\begin{array}{c}20.5 \% \\
(.35)\end{array}$ & $\begin{array}{c}12.2 \% \\
(.25)\end{array}$ & $\begin{array}{c}9 \% \\
(.19)\end{array}$ & $\begin{array}{l}10 \% \\
(.16)\end{array}$ & $\begin{array}{c}14.8 \% \\
(.78)\end{array}$ & $\begin{array}{c}17.6 \% \\
(.91)\end{array}$ & $\begin{array}{l}7.9 \% \\
(.84)\end{array}$ & $\begin{array}{c}10.5 \% \\
(.16)\end{array}$ & $\begin{array}{c}10.7 \% \\
(.61)\end{array}$ \\
\hline Michigan & $\begin{array}{l}8.7 \% \\
(.23)\end{array}$ & $\begin{array}{c}25.1 \% \\
(.43)\end{array}$ & $\begin{array}{l}15 \% \\
(.34)\end{array}$ & $\begin{array}{l}12 \% \\
(.26)\end{array}$ & $\begin{array}{c}12.6 \% \\
(.21)\end{array}$ & $\begin{array}{c}18.2 \% \\
(.81)\end{array}$ & $\begin{array}{l}17.7 \% \\
(1.83)\end{array}$ & $\begin{array}{l}14.3 \% \\
(1.55)\end{array}$ & $\begin{array}{c}13.7 \% \\
(.22)\end{array}$ & $\begin{array}{c}10.6 \% \\
(.65)\end{array}$ \\
\hline Minnesota & $\begin{array}{c}5.9 \% \\
(.2)\end{array}$ & $\begin{array}{c}20.1 \% \\
(.45)\end{array}$ & $\begin{array}{c}11.8 \% \\
(.32)\end{array}$ & $\begin{array}{c}8 \% \\
(.23)\end{array}$ & $\begin{array}{c}9.8 \% \\
(.2)\end{array}$ & $\begin{array}{l}11.9 \% \\
(1.42)\end{array}$ & $\begin{array}{l}12.2 \% \\
(1.68)\end{array}$ & $\begin{array}{l}10.4 \% \\
(1.49)\end{array}$ & $\begin{array}{l}10 \% \\
(.21)\end{array}$ & $\begin{array}{l}7.2 \% \\
(.66)\end{array}$ \\
\hline Mississippi & $\begin{array}{c}11.4 \% \\
(.27)\end{array}$ & $\begin{array}{c}27.7 \% \\
(.48)\end{array}$ & $\begin{array}{c}16.9 \% \\
(.39)\end{array}$ & $\begin{array}{c}15.6 \% \\
(.3)\end{array}$ & $\begin{array}{c}14.3 \% \\
(.27)\end{array}$ & $\begin{array}{c}20.4 \% \\
(.51)\end{array}$ & $\begin{array}{c}16 \% \\
(1.84)\end{array}$ & $\begin{array}{l}16.9 \% \\
(2.21)\end{array}$ & $\begin{array}{c}16.6 \% \\
(.27)\end{array}$ & $\begin{array}{c}14.3 \% \\
(.61)\end{array}$ \\
\hline Missouri & $\begin{array}{l}7.7 \% \\
(.26)\end{array}$ & $\begin{array}{c}22.9 \% \\
(.52)\end{array}$ & $\begin{array}{c}13.7 \% \\
(.39)\end{array}$ & $\begin{array}{c}11.3 \% \\
(.31)\end{array}$ & $\begin{array}{l}12 \% \\
(.26)\end{array}$ & $\begin{array}{l}16 \% \\
(.98)\end{array}$ & $\begin{array}{c}14.2 \% \\
(2.4)\end{array}$ & $\begin{array}{l}13.3 \% \\
(1.65)\end{array}$ & $\begin{array}{c}12.7 \% \\
(.27)\end{array}$ & $\begin{array}{c}10.4 \% \\
(.62)\end{array}$ \\
\hline Montana & $\begin{array}{l}6.5 \% \\
(.21)\end{array}$ & $\begin{array}{c}19.3 \% \\
(.43)\end{array}$ & $\begin{array}{l}11.7 \% \\
(.31)\end{array}$ & $\begin{array}{l}9.3 \% \\
(.26)\end{array}$ & $\begin{array}{c}10.1 \% \\
(.21)\end{array}$ & $\begin{array}{l}19.3 \% \\
(1.97)\end{array}$ & $\begin{array}{l}13.4 \% \\
(1.76)\end{array}$ & $\begin{array}{l}14.3 \% \\
(1.08)\end{array}$ & $\begin{array}{c}10.6 \% \\
(.22)\end{array}$ & $\begin{array}{l}10 \% \\
(.54)\end{array}$ \\
\hline Nebraska & $\begin{array}{l}7.3 \% \\
(.21)\end{array}$ & $\begin{array}{c}22.4 \% \\
(.39)\end{array}$ & $\begin{array}{c}13.3 \% \\
(.31)\end{array}$ & $\begin{array}{c}10.6 \% \\
(.24)\end{array}$ & $\begin{array}{c}11.7 \% \\
(.2)\end{array}$ & $\begin{array}{l}15.7 \% \\
(1.53)\end{array}$ & $\begin{array}{l}16.4 \% \\
(1.39)\end{array}$ & $\begin{array}{r}8.6 \% \\
(1.36)\end{array}$ & $\begin{array}{l}12 \% \\
(.21)\end{array}$ & $\begin{array}{c}11.1 \% \\
(.61)\end{array}$ \\
\hline Nevada & $\begin{array}{l}7.5 \% \\
(.34)\end{array}$ & $\begin{array}{c}23.3 \% \\
(.73)\end{array}$ & $\begin{array}{c}13.9 \% \\
(.5)\end{array}$ & $\begin{array}{c}10.1 \% \\
(.43)\end{array}$ & $\begin{array}{l}12 \% \\
(.38)\end{array}$ & $\begin{array}{l}15.1 \% \\
(1.4)\end{array}$ & $\begin{array}{l}12.9 \% \\
(1.03)\end{array}$ & $\begin{array}{l}8.8 \% \\
(.96)\end{array}$ & $\begin{array}{c}12.2 \% \\
(.35)\end{array}$ & $\begin{array}{c}11.3 \% \\
(.95)\end{array}$ \\
\hline New Hampshire & $\begin{array}{l}6.5 \% \\
(.18)\end{array}$ & $\begin{array}{c}22.2 \% \\
(.43)\end{array}$ & $\begin{array}{c}12.2 \% \\
(.28)\end{array}$ & $\begin{array}{l}9.5 \% \\
(.23)\end{array}$ & $\begin{array}{c}10.7 \% \\
(.19)\end{array}$ & $\begin{array}{l}12.1 \% \\
(1.94)\end{array}$ & $\begin{array}{l}17 \% \\
(2.5)\end{array}$ & $\begin{array}{l}12.2 \% \\
(1.44)\end{array}$ & $\begin{array}{c}11.1 \% \\
(.2)\end{array}$ & $\begin{array}{l}7.9 \% \\
(.48)\end{array}$ \\
\hline New Jersey & $\begin{array}{l}7.9 \% \\
(.18)\end{array}$ & $\begin{array}{c}23.9 \% \\
(.34)\end{array}$ & $\begin{array}{c}14.4 \% \\
(.27)\end{array}$ & $\begin{array}{l}11 \% \\
(.21)\end{array}$ & $\begin{array}{l}11.3 \% \\
(.16)\end{array}$ & $\begin{array}{c}17.5 \% \\
(.58)\end{array}$ & $\begin{array}{l}16.1 \% \\
(.69)\end{array}$ & $\begin{array}{l}10 \% \\
(.88)\end{array}$ & $\begin{array}{c}12.7 \% \\
(.17)\end{array}$ & $\begin{array}{c}12.4 \% \\
(.59)\end{array}$ \\
\hline New Mexico & $\begin{array}{l}8.4 \% \\
(.22)\end{array}$ & $\begin{array}{c}22.3 \% \\
(.42)\end{array}$ & $\begin{array}{c}13.5 \% \\
(.31)\end{array}$ & $\begin{array}{c}11.6 \% \\
(.26)\end{array}$ & $\begin{array}{c}10.2 \% \\
(.23)\end{array}$ & $\begin{array}{c}14.3 \% \\
(.78)\end{array}$ & $\begin{array}{c}16.4 \% \\
(.43)\end{array}$ & $\begin{array}{l}14.3 \% \\
(1.15)\end{array}$ & $\begin{array}{c}12.6 \% \\
(.22)\end{array}$ & $\begin{array}{c}12.3 \% \\
(.49)\end{array}$ \\
\hline New York & $\begin{array}{l}8.5 \% \\
(.23)\end{array}$ & $\begin{array}{c}23.9 \% \\
(.46)\end{array}$ & $\begin{array}{c}14.7 \% \\
(.35)\end{array}$ & $\begin{array}{c}11.5 \% \\
(.27)\end{array}$ & $\begin{array}{c}11.5 \% \\
(.21)\end{array}$ & $\begin{array}{c}18.1 \% \\
(.74)\end{array}$ & $\begin{array}{c}15.6 \% \\
(.86)\end{array}$ & $\begin{array}{l}11.5 \% \\
(1.08)\end{array}$ & $\begin{array}{c}13.3 \% \\
(.23)\end{array}$ & $\begin{array}{c}10.9 \% \\
(.69)\end{array}$ \\
\hline North Carolina & $\begin{array}{l}9.3 \% \\
(.17)\end{array}$ & $\begin{array}{c}25.6 \% \\
(.33)\end{array}$ & $\begin{array}{c}15.4 \% \\
(.26)\end{array}$ & $\begin{array}{c}12.8 \% \\
(.2)\end{array}$ & $\begin{array}{l}13 \% \\
(.18)\end{array}$ & $\begin{array}{c}19.2 \% \\
(.44)\end{array}$ & $\begin{array}{l}11 \% \\
(.73)\end{array}$ & $\begin{array}{l}12 \% \\
(.92)\end{array}$ & $\begin{array}{c}14.3 \% \\
(.17)\end{array}$ & $\begin{array}{c}12.3 \% \\
(.41)\end{array}$ \\
\hline
\end{tabular}


Table 2: Estimated prevalence (sampling standard error)* of total diabetes by state, age, sex, race and insurance status (Figures show actual prevalence; age-standardized figures available from authors). (Continued)

\begin{tabular}{|c|c|c|c|c|c|c|c|c|c|c|}
\hline North Dakota & $\begin{array}{l}6.5 \% \\
(.22)\end{array}$ & $\begin{array}{c}21.7 \% \\
(.49)\end{array}$ & $\begin{array}{c}12.7 \% \\
(.35)\end{array}$ & $\begin{array}{c}10.1 \% \\
(.3)\end{array}$ & $\begin{array}{c}11.1 \% \\
(.23)\end{array}$ & $\begin{array}{l}14.9 \% \\
(2.37)\end{array}$ & $\begin{array}{l}19.1 \% \\
(3.06)\end{array}$ & $\begin{array}{l}15.5 \% \\
(1.83)\end{array}$ & $\begin{array}{c}11.2 \% \\
(.23)\end{array}$ & $\begin{array}{c}12.9 \% \\
(.9)\end{array}$ \\
\hline Ohio & $\begin{array}{l}8.3 \% \\
(.27)\end{array}$ & $\begin{array}{c}24.6 \% \\
(.57)\end{array}$ & $\begin{array}{c}14.6 \% \\
(.4 I)\end{array}$ & $\begin{array}{c}11.9 \% \\
(.33)\end{array}$ & $\begin{array}{c}12.6 \% \\
(.28)\end{array}$ & $\begin{array}{c}18.4 \% \\
(.96)\end{array}$ & $\begin{array}{l}16.5 \% \\
(2.91)\end{array}$ & $\begin{array}{l}14.8 \% \\
(2.05)\end{array}$ & $\begin{array}{c}13.1 \% \\
(.28)\end{array}$ & $\begin{array}{c}13.9 \% \\
(.94)\end{array}$ \\
\hline Oklahoma & $\begin{array}{l}9.7 \% \\
(.22)\end{array}$ & $\begin{array}{c}24.1 \% \\
(.37)\end{array}$ & $\begin{array}{c}15.7 \% \\
(.31)\end{array}$ & $\begin{array}{l}12.9 \% \\
(.24)\end{array}$ & $\begin{array}{c}13.1 \% \\
(.2)\end{array}$ & $\begin{array}{l}18.1 \% \\
(.77)\end{array}$ & $\begin{array}{l}18.3 \% \\
(1.39)\end{array}$ & $\begin{array}{c}17.4 \% \\
(.8)\end{array}$ & $\begin{array}{c}14.8 \% \\
(.22)\end{array}$ & $\begin{array}{c}11.4 \% \\
(.47)\end{array}$ \\
\hline Oregon & $\begin{array}{l}7.1 \% \\
(.21)\end{array}$ & $\begin{array}{c}21.1 \% \\
(.4)\end{array}$ & $\begin{array}{c}13.1 \% \\
(.32)\end{array}$ & $\begin{array}{l}9.6 \% \\
(.23)\end{array}$ & $\begin{array}{c}11.2 \% \\
(.2)\end{array}$ & $\begin{array}{l}13.2 \% \\
(1.14)\end{array}$ & $\begin{array}{l}11.7 \% \\
(1.34)\end{array}$ & $\begin{array}{l}11.2 \% \\
(1.36)\end{array}$ & $\begin{array}{l}11.6 \% \\
(.21)\end{array}$ & $\begin{array}{l}9.0 \% \\
(.56)\end{array}$ \\
\hline Pennsylvania & $\begin{array}{l}8.1 \% \\
(.23)\end{array}$ & $\begin{array}{c}24.1 \% \\
(.43)\end{array}$ & $\begin{array}{c}15.1 \% \\
(.36)\end{array}$ & $\begin{array}{c}11.6 \% \\
(.25)\end{array}$ & $\begin{array}{c}12.7 \% \\
(.21)\end{array}$ & $\begin{array}{c}17.7 \% \\
(.99)\end{array}$ & $\begin{array}{l}19.1 \% \\
(2.16)\end{array}$ & $\begin{array}{l}12.8 \% \\
(1.71)\end{array}$ & $\begin{array}{c}13.6 \% \\
(.23)\end{array}$ & $\begin{array}{c}10.4 \% \\
(.66)\end{array}$ \\
\hline Rhode Island & $\begin{array}{c}7 \% \\
(.23)\end{array}$ & $\begin{array}{c}22.7 \% \\
(.5)\end{array}$ & $\begin{array}{c}13.7 \% \\
(.37)\end{array}$ & $\begin{array}{c}10.3 \% \\
(.29)\end{array}$ & $\begin{array}{c}11.7 \% \\
(.24)\end{array}$ & $\begin{array}{l}15.4 \% \\
(1.39)\end{array}$ & $\begin{array}{l}16.9 \% \\
(1.31)\end{array}$ & $\begin{array}{c}6.7 \% \\
(1.15)\end{array}$ & $\begin{array}{c}12.1 \% \\
(.25)\end{array}$ & $\begin{array}{c}10.3 \% \\
(.78)\end{array}$ \\
\hline South Carolina & $\begin{array}{l}10 \% \\
(.22)\end{array}$ & $\begin{array}{l}26 \% \\
(.4)\end{array}$ & $\begin{array}{c}15.8 \% \\
(.32)\end{array}$ & $\begin{array}{c}13.4 \% \\
(.26)\end{array}$ & $\begin{array}{c}12.6 \% \\
(.2)\end{array}$ & $\begin{array}{c}20.4 \% \\
(.55)\end{array}$ & $\begin{array}{l}18.7 \% \\
(1.91)\end{array}$ & $\begin{array}{l}10.5 \% \\
(1.17)\end{array}$ & $\begin{array}{c}\mid 4.7 \% \\
(.22)\end{array}$ & $\begin{array}{c}14.0 \% \\
(.57)\end{array}$ \\
\hline South Dakota & $\begin{array}{c}7 \% \\
(.19)\end{array}$ & $\begin{array}{c}21.5 \% \\
(.37)\end{array}$ & $\begin{array}{l}13 \% \\
(.28)\end{array}$ & $\begin{array}{c}10.3 \% \\
(.23)\end{array}$ & $\begin{array}{l}11.2 \% \\
(.18)\end{array}$ & $\begin{array}{l}17.7 \% \\
(1.69)\end{array}$ & $\begin{array}{c}16.5 \% \\
(2.2)\end{array}$ & $\begin{array}{l}18.9 \% \\
(1.18)\end{array}$ & $\begin{array}{c}11.7 \% \\
(.19)\end{array}$ & $\begin{array}{c}10.9 \% \\
(.56)\end{array}$ \\
\hline Tennessee & $\begin{array}{c}10.5 \% \\
(.37)\end{array}$ & $\begin{array}{c}26.3 \% \\
(.6)\end{array}$ & $\begin{array}{c}16.3 \% \\
(.53)\end{array}$ & $\begin{array}{c}13.8 \% \\
(.38)\end{array}$ & $\begin{array}{c}14.6 \% \\
(.32)\end{array}$ & $\begin{array}{l}17.9 \% \\
(1.18)\end{array}$ & $\begin{array}{l}13.3 \% \\
(2.41)\end{array}$ & $\begin{array}{l}13.6 \% \\
(2.67)\end{array}$ & $\begin{array}{c}15.4 \% \\
(.35)\end{array}$ & $\begin{array}{c}12.0 \% \\
(.78)\end{array}$ \\
\hline Texas & $\begin{array}{l}10.1 \% \\
(.25)\end{array}$ & $\begin{array}{c}25.4 \% \\
(.46)\end{array}$ & $\begin{array}{c}15.2 \% \\
(.35)\end{array}$ & $\begin{array}{l}13 \% \\
(.28)\end{array}$ & $\begin{array}{c}12.2 \% \\
(.26)\end{array}$ & $\begin{array}{c}17.1 \% \\
(.65)\end{array}$ & $\begin{array}{c}18.6 \% \\
(.6)\end{array}$ & $\begin{array}{c}12 \% \\
(1.17)\end{array}$ & $\begin{array}{c}14.3 \% \\
(.25)\end{array}$ & $\begin{array}{c}13.4 \% \\
(.5)\end{array}$ \\
\hline Utah & $\begin{array}{l}6.3 \% \\
(.22)\end{array}$ & $\begin{array}{c}22.7 \% \\
(.56)\end{array}$ & $\begin{array}{l}12 \% \\
(.35)\end{array}$ & $\begin{array}{l}8.7 \% \\
(.28)\end{array}$ & $\begin{array}{l}10 \% \\
(.23)\end{array}$ & $\begin{array}{c}14 \% \\
(1.96)\end{array}$ & $\begin{array}{l}14 \% \\
(1.4)\end{array}$ & $\begin{array}{l}11.1 \% \\
(1.59)\end{array}$ & $\begin{array}{c}10.6 \% \\
(.24)\end{array}$ & $\begin{array}{l}8.1 \% \\
(.62)\end{array}$ \\
\hline Vermont & $\begin{array}{l}6.1 \% \\
(.16)\end{array}$ & $\begin{array}{c}19.9 \% \\
(.37\end{array}$ & $\begin{array}{c}11.7 \% \\
(.26)\end{array}$ & $\begin{array}{c}8.6 \% \\
(.2)\end{array}$ & $\begin{array}{l}10 \% \\
(.16)\end{array}$ & $\begin{array}{l}13.3 \% \\
(1.65)\end{array}$ & $\begin{array}{l}14.9 \% \\
(2.03)\end{array}$ & $\begin{array}{l}11.8 \% \\
(1.47)\end{array}$ & $\begin{array}{c}10.3 \% \\
(.17)\end{array}$ & $\begin{array}{l}8.4 \% \\
(.49)\end{array}$ \\
\hline Virginia & $\begin{array}{l}7.7 \% \\
(.24)\end{array}$ & $\begin{array}{c}23.6 \% \\
(.58)\end{array}$ & $\begin{array}{c}13.5 \% \\
(.39)\end{array}$ & $\begin{array}{l}10.5 \% \\
(.31)\end{array}$ & $\begin{array}{c}11.3 \% \\
(.26)\end{array}$ & $\begin{array}{c}16.8 \% \\
(.81)\end{array}$ & $\begin{array}{l}11.3 \% \\
(1.15)\end{array}$ & $\begin{array}{c}10 \% \\
(1.49)\end{array}$ & $\begin{array}{l}11.9 \% \\
(.25)\end{array}$ & $\begin{array}{c}12.6 \% \\
(.95)\end{array}$ \\
\hline Washington & $\begin{array}{l}7.4 \% \\
(.12)\end{array}$ & $\begin{array}{c}21.3 \% \\
(.23)\end{array}$ & $\begin{array}{l}12.5 \% \\
(.17)\end{array}$ & $\begin{array}{l}9.9 \% \\
(.13)\end{array}$ & $\begin{array}{l}11.1 \% \\
(.11)\end{array}$ & $\begin{array}{c}13.5 \% \\
(.69)\end{array}$ & $\begin{array}{c}13.4 \% \\
(.64)\end{array}$ & $\begin{array}{c}10.7 \% \\
(.56)\end{array}$ & $\begin{array}{l}11.4 \% \\
(.12)\end{array}$ & $\begin{array}{c}10.0 \% \\
(.35)\end{array}$ \\
\hline West Virginia & $\begin{array}{l}11.1 \% \\
(.31)\end{array}$ & $\begin{array}{c}27.3 \% \\
(.53)\end{array}$ & $\begin{array}{c}17.6 \% \\
(.44)\end{array}$ & $\begin{array}{c}15.4 \% \\
(.36)\end{array}$ & $\begin{array}{c}16.4 \% \\
(.29)\end{array}$ & $\begin{array}{c}19 \% \\
(1.85)\end{array}$ & $\begin{array}{l}15.1 \% \\
(2.14)\end{array}$ & $\begin{array}{l}17.1 \% \\
(1.87)\end{array}$ & $\begin{array}{c}17.2 \% \\
(.31)\end{array}$ & $\begin{array}{c}11.9 \% \\
(.72)\end{array}$ \\
\hline Wisconsin & $\begin{array}{l}6.3 \% \\
(.2)\end{array}$ & $\begin{array}{c}21.6 \% \\
(.49)\end{array}$ & $\begin{array}{l}12.1 \% \\
(.32)\end{array}$ & $\begin{array}{l}9.4 \% \\
(.26)\end{array}$ & $\begin{array}{c}10.4 \% \\
(.21)\end{array}$ & $\begin{array}{l}17.2 \% \\
(1.34)\end{array}$ & $\begin{array}{l}14.6 \% \\
(2.22)\end{array}$ & $\begin{array}{l}10.6 \% \\
(1.64)\end{array}$ & $\begin{array}{c}10.7 \% \\
(.22)\end{array}$ & $\begin{array}{c}11.9 \% \\
(.8)\end{array}$ \\
\hline Wyoming & $\begin{array}{l}7.3 \% \\
(.22)\end{array}$ & $\begin{array}{c}21.2 \% \\
(.43)\end{array}$ & $\begin{array}{l}12.8 \% \\
(.32)\end{array}$ & $\begin{array}{l}9.8 \% \\
(.25)\end{array}$ & $\begin{array}{c}10.8 \% \\
(.2)\end{array}$ & $\begin{array}{l}16.2 \% \\
(2.07)\end{array}$ & $\begin{array}{l}20.4 \% \\
(1.58)\end{array}$ & $\begin{array}{l}15.6 \% \\
(1.86)\end{array}$ & $\begin{array}{l}11.5 \% \\
(.22)\end{array}$ & $\begin{array}{c}10.0 \% \\
(.54)\end{array}$ \\
\hline
\end{tabular}

* The standard error of prevalence reported here reflects the sampling variability in the predicted diabetes prevalence but does not incorporate uncertainty in the prediction model (parameter uncertainty and stochastic uncertainty) and thus is an underestimate for the true standard error of prevalence. We included the parameter and stochastic uncertainty of the modeling using a multiple imputation approach in which we imputed the prevalence of diabetes for individuals who did not report having diabetes 10 times, drawing from a multivariate Normal distribution of the coefficients and drawing randomly from the posterior binomial distribution of revalence. We estimated the standard error of the national prevalence of diabetes using these 10 imputed values for each sex. The standard error was $0.8 \%$ for men and $0.4 \%$ for women, which is almost 10 times larger than the standard error estimated using sampling uncertainty only. 

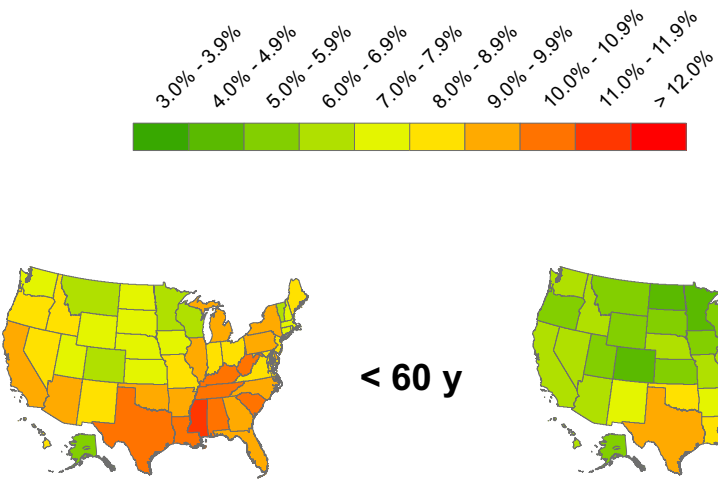

$<60 y$

Male

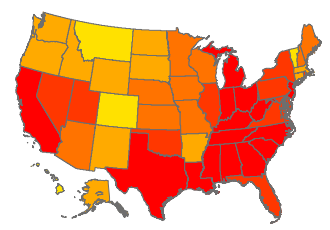

$\geq 60 y$

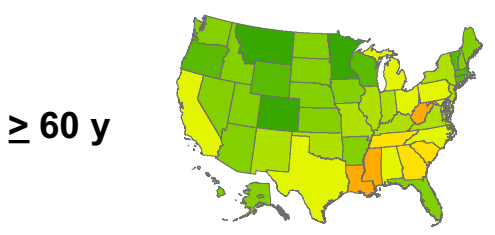

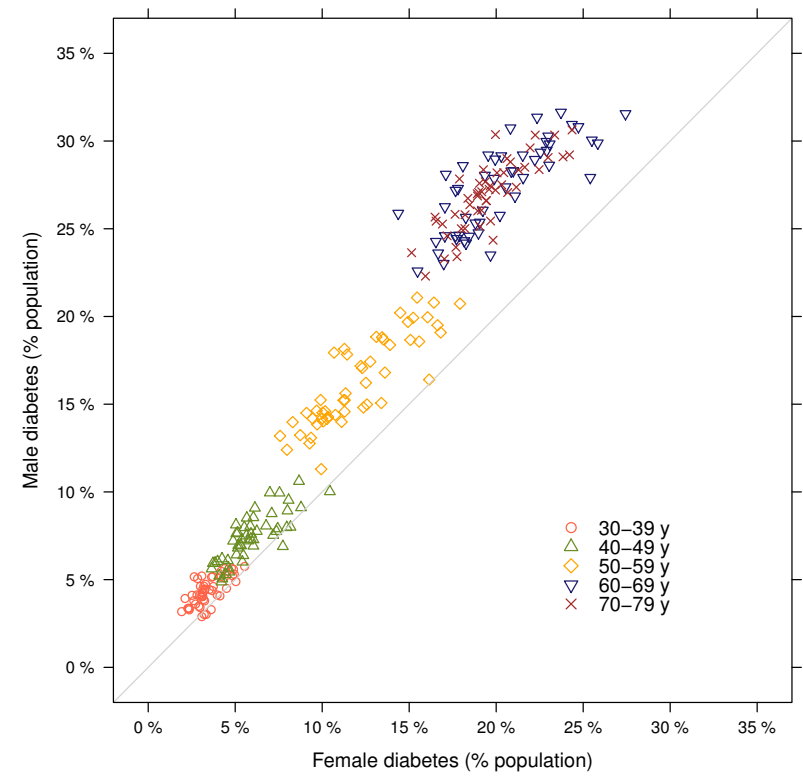

Figure 2

Relationship between male and female diabetes prevalence, by age. Each data point corresponds to one state.

Men in all states had higher proportions of undiagnosed diabetes than women, with the male-female difference in undiagnosed proportion being largest in Hawaii, Mississippi, District of Columbia, West Virginia, and Idaho, where the proportion undiagnosed among men was $34.1 \%$ to $39.0 \%$ higher than among women. The malefemale diagnosis disparity was smallest in Colorado, Pennsylvania, Vermont, and Minnesota (12.9\% to $19.8 \%)$. When stratified on race, the proportion of cases undiagnosed was highest among Hispanics (33\%), followed by whites (28\%) and blacks (19\%), and it was lowest in the residual group of "other races" (6\%). One-third of diabetes cases were undiagnosed in participants who did not have insurance compared to one-fourth among insured Americans.

\section{Discussion}

To our knowledge, this is the first study to estimate the total prevalence of diabetes and the proportion of diabetes that is undiagnosed at the state level. The Southern and Appalachian states had the highest diabetes prevalence, with Mississippi faring the worst. The Northern plains, the Northeast and the Midwest had the lowest prevalence. Prevalence of undiagnosed diabetes also varied across states, with Southern states and California having the highest prevalence. The proportion of undiagnosed diabetes was higher in men, Hispanics, and the uninsured compared to women, whites and insured. In fact, one-half of 
Table 3: Estimated prevalence (sampling standard error)* of undiagnosed diabetes by state, age, sex, race, and insurance (Figures show actual prevalence; age-standardized figures available from authors).

\begin{tabular}{|c|c|c|c|c|c|c|c|c|c|c|}
\hline \multirow[t]{2}{*}{ State } & \multicolumn{2}{|c|}{ Age group } & \multicolumn{2}{|r|}{ Sex } & \multicolumn{4}{|c|}{ Race } & \multicolumn{2}{|c|}{ Insurance status } \\
\hline & $30-59 y$ & $\geq 60 y$ & Men & Women & White & Black & Hispanic & Other races & Insured & Uninsured \\
\hline National NHANES & $\begin{array}{c}2.1 \% \\
(.4)\end{array}$ & $\begin{array}{l}6.7 \% \\
(.9)\end{array}$ & $\begin{array}{c}4.5 \% \\
(.6)\end{array}$ & $\begin{array}{l}2.3 \% \\
(.4)\end{array}$ & $\begin{array}{c}3.4 \% \\
(.5)\end{array}$ & $\begin{array}{c}3.3 \% \\
(.6)\end{array}$ & $\begin{array}{l}4.4 \% \\
(1.1)\end{array}$ & $\begin{array}{l}.5 \% \\
(.5)\end{array}$ & $\begin{array}{c}3.4 \% \\
(.4)\end{array}$ & $\begin{array}{c}3.2 \% \\
(.7)\end{array}$ \\
\hline National BRFSS Prediction & $\begin{array}{c}2.1 \% \\
(<.01)\end{array}$ & $\begin{array}{l}6.4 \% \\
(.01)\end{array}$ & $\begin{array}{l}4.3 \% \\
(0.1)\end{array}$ & $\begin{array}{c}2.5 \% \\
(<.01)\end{array}$ & $\begin{array}{c}3.3 \% \\
(<.01)\end{array}$ & $\begin{array}{l}3.3 \% \\
(.02)\end{array}$ & $\begin{array}{l}5.4 \% \\
(.05)\end{array}$ & $\begin{array}{l}.7 \% \\
(.01)\end{array}$ & $\begin{array}{c}3.3 \% \\
(<.01)\end{array}$ & $\begin{array}{c}3.9 \% \\
(0.02)\end{array}$ \\
\hline Alabama & $\begin{array}{l}2.1 \% \\
(.03)\end{array}$ & $\begin{array}{l}6.2 \% \\
(.09)\end{array}$ & $\begin{array}{l}4.3 \% \\
(.07)\end{array}$ & $\begin{array}{l}2.5 \% \\
(.03)\end{array}$ & $\begin{array}{l}3.4 \% \\
(.04)\end{array}$ & $\begin{array}{l}3.5 \% \\
(.08)\end{array}$ & $\begin{array}{l}5.9 \% \\
(.43)\end{array}$ & $\begin{array}{c}.9 \% \\
(.07)\end{array}$ & $\begin{array}{l}3.3 \% \\
(.04)\end{array}$ & $\begin{array}{l}3.8 \% \\
(.12)\end{array}$ \\
\hline Alaska & $\begin{array}{l}1.9 \% \\
(.04)\end{array}$ & $\begin{array}{l}6.3 \% \\
(.18)\end{array}$ & $\begin{array}{l}3.5 \% \\
(.09)\end{array}$ & $\begin{array}{l}1.9 \% \\
(.05)\end{array}$ & $\begin{array}{c}3 \% \\
(.06)\end{array}$ & $\begin{array}{l}3.5 \% \\
(.21)\end{array}$ & $\begin{array}{l}5.3 \% \\
(.55)\end{array}$ & $\begin{array}{c}.9 \% \\
(.04)\end{array}$ & $\begin{array}{l}2.7 \% \\
(.06)\end{array}$ & $\begin{array}{l}2.9 \% \\
(.13)\end{array}$ \\
\hline Arizona & $\begin{array}{l}2.2 \% \\
(.05)\end{array}$ & $\begin{array}{l}6.3 \% \\
(.12)\end{array}$ & $\begin{array}{l}4.4 \% \\
(.09)\end{array}$ & $\begin{array}{l}2.5 \% \\
(.05)\end{array}$ & $\begin{array}{l}3.4 \% \\
(.05)\end{array}$ & $\begin{array}{l}2.8 \% \\
(.15)\end{array}$ & $\begin{array}{l}5.1 \% \\
(.24)\end{array}$ & $\begin{array}{c}.7 \% \\
(.07)\end{array}$ & $\begin{array}{l}3.4 \% \\
(.06)\end{array}$ & $\begin{array}{c}4 \% \\
(.16)\end{array}$ \\
\hline Arkansas & $\begin{array}{l}2.1 \% \\
(.02)\end{array}$ & $\begin{array}{l}6.2 \% \\
(.07)\end{array}$ & $\begin{array}{l}4.4 \% \\
(.06)\end{array}$ & $\begin{array}{l}2.5 \% \\
(.03)\end{array}$ & $\begin{array}{l}3.5 \% \\
(.03)\end{array}$ & $\begin{array}{l}3.4 \% \\
(.1)\end{array}$ & $\begin{array}{l}5.6 \% \\
(.39)\end{array}$ & $\begin{array}{c}.9 \% \\
(.04)\end{array}$ & $\begin{array}{l}3.4 \% \\
(.03)\end{array}$ & $\begin{array}{l}3.7 \% \\
(.1)\end{array}$ \\
\hline California & $\begin{array}{l}2.4 \% \\
(.04)\end{array}$ & $\begin{array}{l}6.3 \% \\
(.11)\end{array}$ & $\begin{array}{l}4.5 \% \\
(.08)\end{array}$ & $\begin{array}{l}2.5 \% \\
(.04)\end{array}$ & $\begin{array}{l}3.1 \% \\
(.03)\end{array}$ & $\begin{array}{l}3.4 \% \\
(.12)\end{array}$ & $\begin{array}{l}5.3 \% \\
(.13)\end{array}$ & $\begin{array}{l}.7 \% \\
(.03)\end{array}$ & $\begin{array}{l}3.3 \% \\
(.04)\end{array}$ & $\begin{array}{l}4.3 \% \\
(.14)\end{array}$ \\
\hline Colorado & $\begin{array}{l}2 \% \\
(.02)\end{array}$ & $\begin{array}{l}6.2 \% \\
(.07)\end{array}$ & $\begin{array}{l}3.8 \% \\
(.05)\end{array}$ & $\begin{array}{l}2.2 \% \\
(.02)\end{array}$ & $\begin{array}{l}2.8 \% \\
(.03)\end{array}$ & $\begin{array}{l}2.9 \% \\
(.11)\end{array}$ & $\begin{array}{l}5.2 \% \\
(.15)\end{array}$ & $\begin{array}{c}.7 \% \\
(.04)\end{array}$ & $\begin{array}{l}2.9 \% \\
(.03)\end{array}$ & $\begin{array}{l}3.5 \% \\
(.12)\end{array}$ \\
\hline Connecticut & $\begin{array}{c}2 \% \\
(.02)\end{array}$ & $\begin{array}{l}6.4 \% \\
(.07)\end{array}$ & $\begin{array}{l}4.2 \% \\
(.05)\end{array}$ & $\begin{array}{l}2.4 \% \\
(.03)\end{array}$ & $\begin{array}{l}3.3 \% \\
(.03)\end{array}$ & $\begin{array}{l}3.1 \% \\
(.13)\end{array}$ & $\begin{array}{l}5.1 \% \\
(.22)\end{array}$ & $\begin{array}{c}.6 \% \\
(.04)\end{array}$ & $\begin{array}{l}3.2 \% \\
(.03)\end{array}$ & $\begin{array}{l}4.1 \% \\
(.15)\end{array}$ \\
\hline Delaware & $\begin{array}{c}2 \% \\
(.03)\end{array}$ & $\begin{array}{l}6.5 \% \\
(.09)\end{array}$ & $\begin{array}{l}4.3 \% \\
(.08)\end{array}$ & $\begin{array}{l}2.5 \% \\
(.04)\end{array}$ & $\begin{array}{l}3.5 \% \\
(.05)\end{array}$ & $\begin{array}{l}3.2 \% \\
(.12)\end{array}$ & $\begin{array}{l}4.7 \% \\
(.44)\end{array}$ & $\begin{array}{c}.7 \% \\
(.05)\end{array}$ & $\begin{array}{l}3.4 \% \\
(.04)\end{array}$ & $\begin{array}{l}3.5 \% \\
(.18)\end{array}$ \\
\hline District of Columbia & $\begin{array}{l}1.9 \% \\
(.03)\end{array}$ & $\begin{array}{l}6.2 \% \\
(.12)\end{array}$ & $\begin{array}{l}3.8 \% \\
(.08)\end{array}$ & $\begin{array}{l}2.4 \% \\
(.05)\end{array}$ & $\begin{array}{l}2.4 \% \\
(.04)\end{array}$ & $\begin{array}{l}3.5 \% \\
(.07)\end{array}$ & $\begin{array}{c}4.4 \% \\
(.3)\end{array}$ & $\begin{array}{c}.6 \% \\
(.05)\end{array}$ & $\begin{array}{c}3 \% \\
(.05)\end{array}$ & $\begin{array}{l}4 \% \\
(.2)\end{array}$ \\
\hline Florida & $\begin{array}{l}2.3 \% \\
(.03)\end{array}$ & $\begin{array}{l}6.9 \% \\
(.08)\end{array}$ & $\begin{array}{l}5.1 \% \\
(.07)\end{array}$ & $\begin{array}{l}2.9 \% \\
(.04)\end{array}$ & $\begin{array}{l}3.8 \% \\
(.04)\end{array}$ & $\begin{array}{l}3.5 \% \\
(.11)\end{array}$ & $\begin{array}{l}6.2 \% \\
(.18)\end{array}$ & $\begin{array}{c}.8 \% \\
(.05)\end{array}$ & $\begin{array}{l}3.9 \% \\
(.04)\end{array}$ & $\begin{array}{l}4.4 \% \\
(.13)\end{array}$ \\
\hline Georgia & $\begin{array}{c}2 \% \\
(.02)\end{array}$ & $\begin{array}{l}6.1 \% \\
(.08)\end{array}$ & $\begin{array}{l}3.8 \% \\
(.05)\end{array}$ & $\begin{array}{l}2.2 \% \\
(.02)\end{array}$ & $\begin{array}{l}3.1 \% \\
(.03)\end{array}$ & $\begin{array}{c}3 \% \\
(.06)\end{array}$ & $\begin{array}{l}4.7 \% \\
(.32)\end{array}$ & $\begin{array}{c}.7 \% \\
(.04)\end{array}$ & $\begin{array}{l}2.9 \% \\
(.03)\end{array}$ & $\begin{array}{c}3.4 \% \\
(.1)\end{array}$ \\
\hline Hawaii & $\begin{array}{l}1.4 \% \\
(.02)\end{array}$ & $\begin{array}{l}4.2 \% \\
(.08)\end{array}$ & $\begin{array}{l}3.1 \% \\
(.06)\end{array}$ & $\begin{array}{l}1.5 \% \\
(.03)\end{array}$ & $\begin{array}{l}3.4 \% \\
(.06)\end{array}$ & $\begin{array}{c}3.5 \% \\
(.1)\end{array}$ & $\begin{array}{l}4.9 \% \\
(.23)\end{array}$ & $\begin{array}{c}.9 \% \\
(.02)\end{array}$ & $\begin{array}{l}2.3 \% \\
(.03)\end{array}$ & $\begin{array}{l}2.7 \% \\
(.14)\end{array}$ \\
\hline Idaho & $\begin{array}{l}1.9 \% \\
(.02)\end{array}$ & $\begin{array}{l}6.2 \% \\
(.08)\end{array}$ & $\begin{array}{l}4.1 \% \\
(.06)\end{array}$ & $\begin{array}{l}2.2 \% \\
(.03)\end{array}$ & $\begin{array}{l}3.2 \% \\
(.03)\end{array}$ & $\begin{array}{l}3.1 \% \\
(.23)\end{array}$ & $\begin{array}{l}5.2 \% \\
(.31)\end{array}$ & $\begin{array}{c}.7 \% \\
(.04)\end{array}$ & $\begin{array}{l}3.1 \% \\
(.03)\end{array}$ & $\begin{array}{l}3.4 \% \\
(.1)\end{array}$ \\
\hline Illinois & $\begin{array}{l}2.1 \% \\
(.03)\end{array}$ & $\begin{array}{l}6.5 \% \\
(.09)\end{array}$ & $\begin{array}{l}4.2 \% \\
(.07)\end{array}$ & $\begin{array}{l}2.5 \% \\
(.03)\end{array}$ & $\begin{array}{l}3.3 \% \\
(.03)\end{array}$ & $\begin{array}{c}3.2 \% \\
(.1)\end{array}$ & $\begin{array}{l}5.4 \% \\
(.25)\end{array}$ & $\begin{array}{c}.6 \% \\
(.04)\end{array}$ & $\begin{array}{l}3.2 \% \\
(.04)\end{array}$ & $\begin{array}{l}4.0 \% \\
(.15)\end{array}$ \\
\hline Indiana & $\begin{array}{c}2 \% \\
(.02)\end{array}$ & $\begin{array}{l}6.1 \% \\
(.07)\end{array}$ & $\begin{array}{l}4.1 \% \\
(.05)\end{array}$ & $\begin{array}{l}2.4 \% \\
(.03)\end{array}$ & $\begin{array}{l}3.3 \% \\
(.03)\end{array}$ & $\begin{array}{l}3.3 \% \\
(.11)\end{array}$ & $\begin{array}{l}4.8 \% \\
(.27)\end{array}$ & $\begin{array}{c}.8 \% \\
(.05)\end{array}$ & $\begin{array}{l}3.2 \% \\
(.03)\end{array}$ & $\begin{array}{l}3.5 \% \\
(0.1)\end{array}$ \\
\hline lowa & $\begin{array}{c}2 \% \\
(.02)\end{array}$ & $\begin{array}{l}6.6 \% \\
(.07)\end{array}$ & $\begin{array}{l}4.4 \% \\
(.06)\end{array}$ & $\begin{array}{l}2.6 \% \\
(.03)\end{array}$ & $\begin{array}{l}3.5 \% \\
(.03)\end{array}$ & $\begin{array}{l}3.5 \% \\
(.23)\end{array}$ & $\begin{array}{c}6 \% \\
(.48)\end{array}$ & $\begin{array}{c}.7 \% \\
(.06)\end{array}$ & $\begin{array}{l}3.5 \% \\
(.03)\end{array}$ & $\begin{array}{l}4.1 \% \\
(.16)\end{array}$ \\
\hline
\end{tabular}


Table 3: Estimated prevalence (sampling standard error)* of undiagnosed diabetes by state, age, sex, race, and insurance (Figures show actual prevalence; age-standardized figures available from authors). (Continued)

\begin{tabular}{|c|c|c|c|c|c|c|c|c|c|c|}
\hline Kansas & $\begin{array}{c}2 \% \\
(.02)\end{array}$ & $\begin{array}{l}6.2 \% \\
(.06)\end{array}$ & $\begin{array}{l}4.2 \% \\
(.04)\end{array}$ & $\begin{array}{l}2.4 \% \\
(.02)\end{array}$ & $\begin{array}{l}3.3 \% \\
(.03)\end{array}$ & $\begin{array}{l}3.2 \% \\
(.11)\end{array}$ & $\begin{array}{l}4.9 \% \\
(.18)\end{array}$ & $\begin{array}{c}.8 \% \\
(.04)\end{array}$ & $\begin{array}{l}3.3 \% \\
(.02)\end{array}$ & $\begin{array}{l}3.6 \% \\
(.09)\end{array}$ \\
\hline Kentucky & $\begin{array}{l}2.1 \% \\
(.03)\end{array}$ & $\begin{array}{l}6.1 \% \\
(.07)\end{array}$ & $\begin{array}{l}4.2 \% \\
(.06)\end{array}$ & $\begin{array}{l}2.4 \% \\
(.03)\end{array}$ & $\begin{array}{l}3.3 \% \\
(.03)\end{array}$ & $\begin{array}{l}3.3 \% \\
(.15)\end{array}$ & $\begin{array}{l}5.8 \% \\
(.44)\end{array}$ & $\begin{array}{c}.8 \% \\
(.07)\end{array}$ & $\begin{array}{l}3.2 \% \\
(.03)\end{array}$ & $\begin{array}{l}3.4 \% \\
(.09)\end{array}$ \\
\hline Louisiana & $\begin{array}{l}2.2 \% \\
(.03)\end{array}$ & $\begin{array}{l}6.7 \% \\
(.09)\end{array}$ & $\begin{array}{l}4.4 \% \\
(.06)\end{array}$ & $\begin{array}{l}2.6 \% \\
(.03)\end{array}$ & $\begin{array}{l}3.5 \% \\
(.04)\end{array}$ & $\begin{array}{l}3.5 \% \\
(.07)\end{array}$ & $\begin{array}{l}5.4 \% \\
(.36)\end{array}$ & $\begin{array}{c}1 \% \\
(.06)\end{array}$ & $\begin{array}{l}3.4 \% \\
(.04)\end{array}$ & $\begin{array}{l}3.8 \% \\
(.09)\end{array}$ \\
\hline Maine & $\begin{array}{c}2 \% \\
(.02)\end{array}$ & $\begin{array}{l}6.3 \% \\
(.09)\end{array}$ & $\begin{array}{l}4.2 \% \\
(.06)\end{array}$ & $\begin{array}{l}2.4 \% \\
(.03)\end{array}$ & $\begin{array}{l}3.3 \% \\
(.04)\end{array}$ & $\begin{array}{l}3.1 \% \\
(.42)\end{array}$ & $\begin{array}{l}5.5 \% \\
(.59)\end{array}$ & $\begin{array}{c}.9 \% \\
(.07)\end{array}$ & $\begin{array}{l}3.3 \% \\
(.04)\end{array}$ & $\begin{array}{l}3.8 \% \\
(.14)\end{array}$ \\
\hline Maryland & $\begin{array}{c}2 \% \\
(.02)\end{array}$ & $\begin{array}{l}6.4 \% \\
(.08)\end{array}$ & $\begin{array}{l}4.1 \% \\
(.06)\end{array}$ & $\begin{array}{l}2.3 \% \\
(.03)\end{array}$ & $\begin{array}{l}3.3 \% \\
(.03)\end{array}$ & $\begin{array}{l}3.3 \% \\
(.07)\end{array}$ & $\begin{array}{l}4.8 \% \\
(.32)\end{array}$ & $\begin{array}{l}.7 \% \\
(.06)\end{array}$ & $\begin{array}{l}3.1 \% \\
(.03)\end{array}$ & $\begin{array}{l}3.7 \% \\
(.14)\end{array}$ \\
\hline Massachusetts & $\begin{array}{l}1.9 \% \\
(.02)\end{array}$ & $\begin{array}{l}6.3 \% \\
(.06)\end{array}$ & $\begin{array}{l}4.1 \% \\
(.05)\end{array}$ & $\begin{array}{l}2.3 \% \\
(.02)\end{array}$ & $\begin{array}{l}3.2 \% \\
(.03)\end{array}$ & $\begin{array}{c}3 \% \\
(.13)\end{array}$ & $\begin{array}{l}4.9 \% \\
(.19)\end{array}$ & $\begin{array}{c}.6 \% \\
(.03)\end{array}$ & $\begin{array}{l}3.1 \% \\
(.03)\end{array}$ & $\begin{array}{l}3.9 \% \\
(.15)\end{array}$ \\
\hline Michigan & $\begin{array}{c}2 \% \\
(.02)\end{array}$ & $\begin{array}{l}6.4 \% \\
(.07)\end{array}$ & $\begin{array}{l}4.2 \% \\
(.05)\end{array}$ & $\begin{array}{l}2.4 \% \\
(.03)\end{array}$ & $\begin{array}{l}3.4 \% \\
(.03)\end{array}$ & $\begin{array}{l}3.2 \% \\
(.09)\end{array}$ & $\begin{array}{l}5.6 \% \\
(.46)\end{array}$ & $\begin{array}{c}.7 \% \\
(.04)\end{array}$ & $\begin{array}{l}3.3 \% \\
(.03)\end{array}$ & $\begin{array}{l}3.4 \% \\
(.11)\end{array}$ \\
\hline Minnesota & $\begin{array}{l}1.9 \% \\
(.02)\end{array}$ & $\begin{array}{l}6.6 \% \\
(.08)\end{array}$ & $\begin{array}{l}4.1 \% \\
(.06)\end{array}$ & $\begin{array}{l}2.4 \% \\
(.03)\end{array}$ & $\begin{array}{l}3.3 \% \\
(.03)\end{array}$ & $\begin{array}{l}2.9 \% \\
(.17)\end{array}$ & $\begin{array}{c}6 \% \\
(.51)\end{array}$ & $\begin{array}{c}.6 \% \\
(.05)\end{array}$ & $\begin{array}{l}3.2 \% \\
(.03)\end{array}$ & $\begin{array}{l}3.6 \% \\
(.15)\end{array}$ \\
\hline Mississippi & $\begin{array}{l}2.1 \% \\
(.02)\end{array}$ & $\begin{array}{l}6.1 \% \\
(.08)\end{array}$ & $\begin{array}{l}4.3 \% \\
(.06)\end{array}$ & $\begin{array}{l}2.4 \% \\
(.03)\end{array}$ & $\begin{array}{l}3.3 \% \\
(.04)\end{array}$ & $\begin{array}{l}3.3 \% \\
(.06)\end{array}$ & $\begin{array}{l}6.5 \% \\
(.47)\end{array}$ & $\begin{array}{c}.8 \% \\
(.07)\end{array}$ & $\begin{array}{l}3.3 \% \\
(.03)\end{array}$ & $\begin{array}{l}3.6 \% \\
(0.1)\end{array}$ \\
\hline Missouri & $\begin{array}{c}2 \% \\
(.03)\end{array}$ & $\begin{array}{l}6.2 \% \\
(.09)\end{array}$ & $\begin{array}{l}4.2 \% \\
(.07)\end{array}$ & $\begin{array}{l}2.4 \% \\
(.03)\end{array}$ & $\begin{array}{l}3.4 \% \\
(.04)\end{array}$ & $\begin{array}{l}3.6 \% \\
(.15)\end{array}$ & $\begin{array}{l}4.8 \% \\
(.37)\end{array}$ & $\begin{array}{c}.8 \% \\
(.06)\end{array}$ & $\begin{array}{l}3.3 \% \\
(.04)\end{array}$ & $\begin{array}{l}3.5 \% \\
(.13)\end{array}$ \\
\hline Montana & $\begin{array}{c}2 \% \\
(.02)\end{array}$ & $\begin{array}{l}6.3 \% \\
(.08)\end{array}$ & $\begin{array}{l}4.2 \% \\
(.06)\end{array}$ & $\begin{array}{l}2.4 \% \\
(.03)\end{array}$ & $\begin{array}{l}3.4 \% \\
(.04)\end{array}$ & $\begin{array}{c}3 \% \\
(.19)\end{array}$ & $\begin{array}{l}5.1 \% \\
(.38)\end{array}$ & $\begin{array}{c}.8 \% \\
(.03)\end{array}$ & $\begin{array}{l}3.3 \% \\
(.04)\end{array}$ & $\begin{array}{l}3.4 \% \\
(.09)\end{array}$ \\
\hline Nebraska & $\begin{array}{c}2 \% \\
(.02)\end{array}$ & $\begin{array}{l}6.4 \% \\
(.06)\end{array}$ & $\begin{array}{l}4.3 \% \\
(.05)\end{array}$ & $\begin{array}{l}2.5 \% \\
(.03)\end{array}$ & $\begin{array}{l}3.4 \% \\
(.03)\end{array}$ & $\begin{array}{l}3.2 \% \\
(.16)\end{array}$ & $\begin{array}{l}5.3 \% \\
(.28)\end{array}$ & $\begin{array}{c}.8 \% \\
(.06)\end{array}$ & $\begin{array}{l}3.3 \% \\
(.03)\end{array}$ & $\begin{array}{l}3.9 \% \\
(.11)\end{array}$ \\
\hline Nevada & $\begin{array}{l}2.1 \% \\
(.04)\end{array}$ & $\begin{array}{l}6.3 \% \\
(.13)\end{array}$ & $\begin{array}{l}4.3 \% \\
(.09)\end{array}$ & $\begin{array}{l}2.3 \% \\
(.05)\end{array}$ & $\begin{array}{l}3.4 \% \\
(.06)\end{array}$ & $\begin{array}{l}3.3 \% \\
(.18)\end{array}$ & $\begin{array}{l}5.4 \% \\
(.26)\end{array}$ & $\begin{array}{c}.7 \% \\
(.04)\end{array}$ & $\begin{array}{l}3.3 \% \\
(.05)\end{array}$ & $\begin{array}{l}3.8 \% \\
(.19)\end{array}$ \\
\hline New Hampshire & $\begin{array}{l}1.9 \% \\
(.02)\end{array}$ & $\begin{array}{l}6.5 \% \\
(.07)\end{array}$ & $\begin{array}{l}4.1 \% \\
(.05)\end{array}$ & $\begin{array}{l}2.3 \% \\
(.03)\end{array}$ & $\begin{array}{l}3.2 \% \\
(.03)\end{array}$ & $\begin{array}{l}2.8 \% \\
(.24)\end{array}$ & $\begin{array}{l}5.3 \% \\
(.54)\end{array}$ & $\begin{array}{l}.8 \% \\
(.06)\end{array}$ & $\begin{array}{l}3.2 \% \\
(.03)\end{array}$ & $\begin{array}{l}3.2 \% \\
(.11)\end{array}$ \\
\hline New Jersey & $\begin{array}{l}2.2 \% \\
(.02)\end{array}$ & $\begin{array}{l}6.6 \% \\
(.07)\end{array}$ & $\begin{array}{l}4.5 \% \\
(.05)\end{array}$ & $\begin{array}{l}2.6 \% \\
(.02)\end{array}$ & $\begin{array}{l}3.4 \% \\
(.03)\end{array}$ & $\begin{array}{l}3.5 \% \\
(.08)\end{array}$ & $\begin{array}{l}5.7 \% \\
(.18)\end{array}$ & $\begin{array}{c}.6 \% \\
(.03)\end{array}$ & $\begin{array}{l}3.4 \% \\
(.03)\end{array}$ & $\begin{array}{l}4.5 \% \\
(.16)\end{array}$ \\
\hline New Mexico & $\begin{array}{l}2.6 \% \\
(.03)\end{array}$ & $\begin{array}{c}7 \% \\
(.09)\end{array}$ & $\begin{array}{l}4.9 \% \\
(.07)\end{array}$ & $\begin{array}{l}2.9 \% \\
(.03)\end{array}$ & $\begin{array}{l}3.3 \% \\
(.04)\end{array}$ & $\begin{array}{c}3.4 \% \\
(.1)\end{array}$ & $\begin{array}{c}5.7 \% \\
(.1)\end{array}$ & $\begin{array}{c}.8 \% \\
(.03)\end{array}$ & $\begin{array}{l}3.8 \% \\
(.04)\end{array}$ & $\begin{array}{l}4.4 \% \\
(.12)\end{array}$ \\
\hline New York & $\begin{array}{l}2.1 \% \\
(.03)\end{array}$ & $\begin{array}{l}6.6 \% \\
(.08)\end{array}$ & $\begin{array}{l}4.4 \% \\
(.06)\end{array}$ & $\begin{array}{l}2.6 \% \\
(.03)\end{array}$ & $\begin{array}{l}3.4 \% \\
(.03)\end{array}$ & $\begin{array}{l}3.3 \% \\
(.09)\end{array}$ & $\begin{array}{l}5.8 \% \\
(.21)\end{array}$ & $\begin{array}{c}.8 \% \\
(.04)\end{array}$ & $\begin{array}{l}3.4 \% \\
(.04)\end{array}$ & $\begin{array}{l}4.1 \% \\
(.16)\end{array}$ \\
\hline North Carolina & $\begin{array}{l}2.1 \% \\
(.02)\end{array}$ & $\begin{array}{l}6.2 \% \\
(.05)\end{array}$ & $\begin{array}{l}4.2 \% \\
(.04)\end{array}$ & $\begin{array}{l}2.4 \% \\
(.02)\end{array}$ & $\begin{array}{l}3.3 \% \\
(.02)\end{array}$ & $\begin{array}{l}3.6 \% \\
(.06)\end{array}$ & $\begin{array}{l}5.2 \% \\
(.24)\end{array}$ & $\begin{array}{c}.8 \% \\
(.03)\end{array}$ & $\begin{array}{l}3.2 \% \\
(.02)\end{array}$ & $\begin{array}{l}3.8 \% \\
(.08)\end{array}$ \\
\hline North Dakota & $\begin{array}{l}2.1 \% \\
(.03)\end{array}$ & $\begin{array}{l}6.7 \% \\
(.09)\end{array}$ & $\begin{array}{l}4.5 \% \\
(.07)\end{array}$ & $\begin{array}{l}2.6 \% \\
(.04)\end{array}$ & $\begin{array}{l}3.6 \% \\
(.04)\end{array}$ & $\begin{array}{l}3.4 \% \\
(.29)\end{array}$ & $\begin{array}{l}7.6 \% \\
(.73)\end{array}$ & $\begin{array}{c}.9 \% \\
(.07)\end{array}$ & $\begin{array}{l}3.5 \% \\
(.04)\end{array}$ & $\begin{array}{l}3.8 \% \\
(.15)\end{array}$ \\
\hline Ohio & $\begin{array}{c}2 \% \\
(.03)\end{array}$ & $\begin{array}{c}6.5 \% \\
(.1)\end{array}$ & $\begin{array}{l}4.3 \% \\
(.07)\end{array}$ & $\begin{array}{l}2.5 \% \\
(.04)\end{array}$ & $\begin{array}{l}3.4 \% \\
(.04)\end{array}$ & $\begin{array}{l}3.4 \% \\
(.12)\end{array}$ & $\begin{array}{l}6.3 \% \\
(.68)\end{array}$ & $\begin{array}{c}.8 \% \\
(.06)\end{array}$ & $\begin{array}{l}3.4 \% \\
(.04)\end{array}$ & $\begin{array}{l}3.6 \% \\
(.14)\end{array}$ \\
\hline
\end{tabular}


Table 3: Estimated prevalence (sampling standard error)* of undiagnosed diabetes by state, age, sex, race, and insurance (Figures show actual prevalence; age-standardized figures available from authors). (Continued)

\begin{tabular}{|c|c|c|c|c|c|c|c|c|c|c|}
\hline Oklahoma & $\begin{array}{c}2 \% \\
(.02)\end{array}$ & $\begin{array}{l}5.9 \% \\
(.06)\end{array}$ & $\begin{array}{l}4.1 \% \\
(.05)\end{array}$ & $\begin{array}{l}2.4 \% \\
(.02)\end{array}$ & $\begin{array}{l}3.4 \% \\
(.03)\end{array}$ & $\begin{array}{l}3.4 \% \\
(.09)\end{array}$ & $\begin{array}{l}5.8 \% \\
(.31)\end{array}$ & $\begin{array}{c}.9 \% \\
(.03)\end{array}$ & $\begin{array}{l}3.2 \% \\
(.03)\end{array}$ & $\begin{array}{l}3.3 \% \\
(.08)\end{array}$ \\
\hline Oregon & $\begin{array}{l}1.9 \% \\
(.02)\end{array}$ & $\begin{array}{l}6.1 \% \\
(.07)\end{array}$ & $\begin{array}{l}4.1 \% \\
(.05)\end{array}$ & $\begin{array}{l}2.3 \% \\
(.02)\end{array}$ & $\begin{array}{l}3.3 \% \\
(.03)\end{array}$ & $\begin{array}{c}3 \% \\
(.14)\end{array}$ & $\begin{array}{l}4.1 \% \\
(.23)\end{array}$ & $\begin{array}{c}.8 \% \\
(.04)\end{array}$ & $\begin{array}{l}3.1 \% \\
(.03)\end{array}$ & $\begin{array}{l}3.4 \% \\
(0.1)\end{array}$ \\
\hline Pennsylvania & $\begin{array}{c}2 \% \\
(.02)\end{array}$ & $\begin{array}{l}6.4 \% \\
(.07)\end{array}$ & $\begin{array}{l}4.3 \% \\
(.06)\end{array}$ & $\begin{array}{l}2.6 \% \\
(.03)\end{array}$ & $\begin{array}{l}3.5 \% \\
(.03)\end{array}$ & $\begin{array}{l}3.4 \% \\
(.12)\end{array}$ & $\begin{array}{l}5.1 \% \\
(.38)\end{array}$ & $\begin{array}{c}.7 \% \\
(.06)\end{array}$ & $\begin{array}{l}3.4 \% \\
(.03)\end{array}$ & $\begin{array}{l}3.7 \% \\
(.14)\end{array}$ \\
\hline Rhode Island & $\begin{array}{l}2.1 \% \\
(.03)\end{array}$ & $\begin{array}{l}6.5 \% \\
(.08)\end{array}$ & $\begin{array}{l}4.4 \% \\
(.07)\end{array}$ & $\begin{array}{l}2.6 \% \\
(.03)\end{array}$ & $\begin{array}{l}3.5 \% \\
(.04)\end{array}$ & $\begin{array}{l}2.9 \% \\
(.17)\end{array}$ & $\begin{array}{l}5.2 \% \\
(.26)\end{array}$ & $\begin{array}{c}.8 \% \\
(.06)\end{array}$ & $\begin{array}{l}3.4 \% \\
(.04)\end{array}$ & $\begin{array}{l}4.3 \% \\
(.17)\end{array}$ \\
\hline South Carolina & $\begin{array}{l}2.1 \% \\
(.02)\end{array}$ & $\begin{array}{l}6.1 \% \\
(.06)\end{array}$ & $\begin{array}{l}4.1 \% \\
(.05)\end{array}$ & $\begin{array}{l}2.4 \% \\
(.02)\end{array}$ & $\begin{array}{l}3.3 \% \\
(.03)\end{array}$ & $\begin{array}{l}3.3 \% \\
(.06)\end{array}$ & $\begin{array}{l}5.5 \% \\
(.4 I)\end{array}$ & $\begin{array}{c}.8 \% \\
(.05)\end{array}$ & $\begin{array}{l}3.2 \% \\
(.03)\end{array}$ & $\begin{array}{l}3.6 \% \\
(.08)\end{array}$ \\
\hline South Dakota & $\begin{array}{c}2 \% \\
(.02)\end{array}$ & $\begin{array}{l}6.4 \% \\
(.06)\end{array}$ & $\begin{array}{l}4.4 \% \\
(.05)\end{array}$ & $\begin{array}{l}2.5 \% \\
(.03)\end{array}$ & $\begin{array}{l}3.5 \% \\
(.03)\end{array}$ & $\begin{array}{l}3.5 \% \\
(.24)\end{array}$ & $\begin{array}{c}6.4 \% \\
(.5)\end{array}$ & $\begin{array}{c}.8 \% \\
(.03)\end{array}$ & $\begin{array}{l}3.4 \% \\
(.03)\end{array}$ & $\begin{array}{l}3.7 \% \\
(.12)\end{array}$ \\
\hline Tennessee & $\begin{array}{l}2.1 \% \\
(.03)\end{array}$ & $\begin{array}{l}6.1 \% \\
(.09)\end{array}$ & $\begin{array}{l}4.1 \% \\
(.07)\end{array}$ & $\begin{array}{l}2.4 \% \\
(.03)\end{array}$ & $\begin{array}{l}3.3 \% \\
(.04)\end{array}$ & $\begin{array}{l}3.2 \% \\
(.12)\end{array}$ & $\begin{array}{c}5.6 \% \\
(.5)\end{array}$ & $\begin{array}{c}.7 \% \\
(.07)\end{array}$ & $\begin{array}{l}3.2 \% \\
(.04)\end{array}$ & $\begin{array}{l}3.7 \% \\
(.13)\end{array}$ \\
\hline Texas & $\begin{array}{l}2.5 \% \\
(.03)\end{array}$ & $\begin{array}{l}6.7 \% \\
(.09)\end{array}$ & $\begin{array}{l}4.5 \% \\
(.06)\end{array}$ & $\begin{array}{l}2.6 \% \\
(.03)\end{array}$ & $\begin{array}{l}3.3 \% \\
(.03)\end{array}$ & $\begin{array}{l}3.2 \% \\
(.09)\end{array}$ & $\begin{array}{l}5.4 \% \\
(.11)\end{array}$ & $\begin{array}{c}.7 \% \\
(.04)\end{array}$ & $\begin{array}{l}3.4 \% \\
(.04)\end{array}$ & $\begin{array}{l}4.3 \% \\
(.09)\end{array}$ \\
\hline Utah & $\begin{array}{l}1.8 \% \\
(.02)\end{array}$ & $\begin{array}{c}6 \% \\
(.09)\end{array}$ & $\begin{array}{l}3.6 \% \\
(.05)\end{array}$ & $\begin{array}{l}2.1 \% \\
(.03)\end{array}$ & $\begin{array}{l}2.8 \% \\
(.03)\end{array}$ & $\begin{array}{l}2.8 \% \\
(.19)\end{array}$ & $\begin{array}{l}4.6 \% \\
(.22)\end{array}$ & $\begin{array}{c}.6 \% \\
(.05)\end{array}$ & $\begin{array}{l}2.8 \% \\
(.03)\end{array}$ & $\begin{array}{l}3.1 \% \\
(.11)\end{array}$ \\
\hline Vermont & $\begin{array}{l}1.9 \% \\
(.02)\end{array}$ & $\begin{array}{l}6.3 \% \\
(.06)\end{array}$ & $\begin{array}{c}4 \% \\
(.04)\end{array}$ & $\begin{array}{l}2.3 \% \\
(.02)\end{array}$ & $\begin{array}{l}3.2 \% \\
(.03)\end{array}$ & $\begin{array}{l}3.7 \% \\
(.26)\end{array}$ & $\begin{array}{l}5.5 \% \\
(.43)\end{array}$ & $\begin{array}{c}.9 \% \\
(.06)\end{array}$ & $\begin{array}{l}3.1 \% \\
(.03)\end{array}$ & $\begin{array}{c}3.5 \% \\
(.1)\end{array}$ \\
\hline Virginia & $\begin{array}{l}1.9 \% \\
(.03)\end{array}$ & $\begin{array}{l}6.2 \% \\
(.09)\end{array}$ & $\begin{array}{l}3.9 \% \\
(.06)\end{array}$ & $\begin{array}{l}2.3 \% \\
(.03)\end{array}$ & $\begin{array}{l}3.2 \% \\
(.04)\end{array}$ & $\begin{array}{l}3.2 \% \\
(.09)\end{array}$ & $\begin{array}{l}5.4 \% \\
(.35)\end{array}$ & $\begin{array}{c}.7 \% \\
(.05)\end{array}$ & $\begin{array}{c}3 \% \\
(.04)\end{array}$ & $\begin{array}{l}3.7 \% \\
(.14)\end{array}$ \\
\hline Washington & $\begin{array}{l}1.9 \% \\
(.01)\end{array}$ & $\begin{array}{l}6.1 \% \\
(.04)\end{array}$ & $\begin{array}{l}3.9 \% \\
(.03)\end{array}$ & $\begin{array}{l}2.2 \% \\
(.01)\end{array}$ & $\begin{array}{l}3.1 \% \\
(.02)\end{array}$ & $\begin{array}{l}2.9 \% \\
(.08)\end{array}$ & $\begin{array}{l}4.6 \% \\
(.13)\end{array}$ & $\begin{array}{c}.7 \% \\
(.02)\end{array}$ & $\begin{array}{c}3 \% \\
(.02)\end{array}$ & $\begin{array}{l}3.3 \% \\
(.06)\end{array}$ \\
\hline West Virginia & $\begin{array}{l}2.2 \% \\
(.03)\end{array}$ & $\begin{array}{l}6.2 \% \\
(.08)\end{array}$ & $\begin{array}{l}4.5 \% \\
(.07)\end{array}$ & $\begin{array}{l}2.6 \% \\
(.03)\end{array}$ & $\begin{array}{l}3.6 \% \\
(.04)\end{array}$ & $\begin{array}{l}3.8 \% \\
(.23)\end{array}$ & $\begin{array}{l}6.3 \% \\
(.58)\end{array}$ & $\begin{array}{c}.9 \% \\
(.06)\end{array}$ & $\begin{array}{l}3.6 \% \\
(.04)\end{array}$ & $\begin{array}{l}3.3 \% \\
(0.1)\end{array}$ \\
\hline Wisconsin & $\begin{array}{l}1.9 \% \\
(.02)\end{array}$ & $\begin{array}{l}6.5 \% \\
(.08)\end{array}$ & $\begin{array}{l}4.2 \% \\
(.06)\end{array}$ & $\begin{array}{l}2.4 \% \\
(.03)\end{array}$ & $\begin{array}{l}3.3 \% \\
(.04)\end{array}$ & $\begin{array}{l}3.2 \% \\
(.14)\end{array}$ & $\begin{array}{c}4.9 \% \\
(.4)\end{array}$ & $\begin{array}{c}.8 \% \\
(.07)\end{array}$ & $\begin{array}{l}3.2 \% \\
(.04)\end{array}$ & $\begin{array}{l}4.0 \% \\
(.15)\end{array}$ \\
\hline Wyoming & $\begin{array}{l}2.1 \% \\
(.02)\end{array}$ & $\begin{array}{l}6.5 \% \\
(.08)\end{array}$ & $\begin{array}{l}4.3 \% \\
(.06)\end{array}$ & $\begin{array}{l}2.4 \% \\
(.03)\end{array}$ & $\begin{array}{l}3.4 \% \\
(.03)\end{array}$ & $\begin{array}{l}3.5 \% \\
(.24)\end{array}$ & $\begin{array}{c}5.7 \% \\
(.3)\end{array}$ & $\begin{array}{c}.9 \% \\
(.05)\end{array}$ & $\begin{array}{l}3.3 \% \\
(.03)\end{array}$ & $\begin{array}{l}3.6 \% \\
(.11)\end{array}$ \\
\hline
\end{tabular}

* The standard error of prevalence reported here reflects the sampling variability in the predicted diabetes prevalence but does not incorporate uncertainty in the prediction model (parameter uncertainty and stochastic uncertainty) and thus is an underestimate for the true standard error of prevalence. See footnote to Table 2 for an example of how the inclusion of these sources would affect standard errors.

diabetes cases were undiagnosed in uninsured Hispanic men. These findings are important for the development and implementation of adequate state programs to prevent, diagnose, and control diabetes.

This analysis has a number of limitations: First, although our regression models included important sociodemographic, lifestyle, and health system determinants of dia- betes risk and diagnosis, there are other factors that affect diabetes, such as diet and quality of care $[9,10]$. For instance, we were unable to include family history of diabetes, physical activity, alcohol use and specific dietary risk factors of diabetes [11-14] in the model because BRFSS does not include a sufficiently detailed dietary questionnaire or any questions on family history of diabetes and because the questions used to measure alcohol use 
and physical activity are different from those used in NHANES. The effects of some such factors may be captured by the variables in our model (e.g., self-reported diabetes, BMI, smoking, insurance status, visit to a doctor, etc.). If the unexplained effects vary systematically across states, the model may underestimate cross-state variation in diabetes prevalence, making our results conservative. Second, we conducted our analysis using FPG because of its availability for the most recent rounds of NHANES and because it is used by the American Diabetes Association to define diabetes. Other definitions of diabetes, e.g., based on glucose tolerance test, may have led to slightly different estimates. Third, BRFSS response rate varies across states. This may affect the state comparisons if the determinants of non-response are associated with diabetes prevalence. The single best way to reduce uncertainty in our analysis would be the addition of a validation component to BRFSS, which includes measured blood glucose for a random sample of interviewees. Finally, because $50.2 \%$ of observations in NHANES were missing either smoking or insurance status, we used a missing indicator in our regression models to include these observations. Dropping these observations would decrease the precision of our regression coefficients but would not affect the predictions of diabetes prevalence by states materially.

Despite uncertainties, our results currently provide the only estimates of total diabetes and undiagnosed diabetes in U.S. states, and should provide motivation, guidance, and benchmarks for designing, implementing, and evaluating diabetes prevention and control programs at the state level. Further, our methods allow states to combine the relatively low-cost BRFSS telephone survey with NHANES to regularly monitor the prevalence of diabetes and progress in diabetes diagnosis.

Increasing the coverage of lifestyle, e.g., physical activity and pharmacological interventions for diabetes, should be a priority in states with high diabetes prevalence. Some states also need to improve diagnosis, especially among men, because early diagnosis and intensive glycemic control reduces the future incidence of microvascular complications $[15,16]$. Further, diabetes diagnosis will facilitate interventions that lower blood pressure and cholesterol, and hence the risk of cardiovascular disease, among diabetics $[17,18]$. The states with the highest estimated diabetes prevalence in our analysis also have the highest levels of blood pressure and cardiovascular disease risk [19,20]. This geographical distribution of cardiovascular risks and diabetes points to the need for lifestyle and health care interventions that reduce blood pressure and other cardiovascular risks in high-diabetes states.

\section{Competing interests}

The authors declare that they have no competing interests.

\section{Authors' contributions}

GD, CJM and ME designed the study. GD, ABF and SO conducted the analyses. GD and ME wrote the paper with input from other authors. ME oversaw the research and acts as the paper's guarantor. All authors have read and confirmed the final manuscript.

\section{Acknowledgements}

This research was supported by a cooperative agreement from the Centers for Disease Control and Prevention (CDC) through the Association of Schools of Public Health (Grant No. U36/CCU300430-23). The contents of this article are solely the responsibility of the authors and do not necessarily represent the official views of CDC or ASPH. We thank Ali Mokdad for valuable comments on earlier drafts.

\section{References}

I. Murray CJ, Dias RH, Kulkarni SC, Lozano R, Stevens GA, Ezzati M: Improving the comparability of diabetes mortality statistics in the U.S. and Mexico. Diabetes Care 2008, 31 : 45 I-458.

2. Ezzati M, Martin H, Skjold S, Vander HS, Murray CJ: Trends in national and state-level obesity in the USA after correction for self-report bias: analysis of health surveys. J $R$ Soc Med 2006, 99:250-257.

3. Centers for Disease Control and Prevention NCfHS: National Health and Nutrition Examination Survey data sets and related documentation (Survey Questionnaire, Examination and Laboratory Protocols, 1988-1994 and 1999-2002). 2002.

4. Cowie CC, Rust KF, Ford ES, Eberhardt MS, Byrd-Holt DD, Li C, Williams DE, Gregg EW, Bainbridge KE, Saydah SH, Geiss LS: Full accounting of diabetes and pre-diabetes in the U.S. population in 1988-1994 and 2005-2006. Diabetes Care 2009, 32:287-294.

5. Nelson DE, Holtzman D, Bolen J, Stanwyck CA, Mack KA: Reliability and validity of measures from the Behavioral Risk Factor Surveillance System (BRFSS). Soz Praventivmed 200I, 46(Suppl I):S3-42.

6. Remington PL, Smith MY, Williamson DF, Anda RF, Gentry EM, Hogelin GC: Design, characteristics, and usefulness of state-based behavioral risk factor surveillance: 1981-87. Public Health Rep 1988, 103:366-375.

7. Screening for type 2 diabetes mellitus in adults: recommendations and rationale. Am J Nurs 2004, 104:83-90.

8. Danaei G, Lawes CM, Vander HS, Murray C], Ezzati M: Global and regional mortality from ischaemic heart disease and stroke attributable to higher-thanoptimum blood glucose concentration: comparative risk assessment. Lancet 2006, 368:165I-1659.

9. Hu FB, Manson JE, Stampfer MJ, Colditz G, Liu S, Solomon CG, Willett WC: Diet, lifestyle, and the risk of type 2 diabetes mellitus in women. N Engl J Med 200I, 345:790-797.

10. Trivedi AN, Zaslavsky AM, Schneider EC, Ayanian JZ: Trends in the quality of care and racial disparities in Medicare managed care. N Engl J Med 2005, 353:692-700.

II. Hu FB, Leitzmann MF, Stampfer MJ, Colditz GA, Willett WC, Rimm EB: Physical activity and television watching in relation to risk for type 2 diabetes mellitus in men. Arch Intern Med 200I, 161:1542-1548.

12. Hu FB, Li TY, Colditz GA, Willett WC, Manson JE: Television watching and other sedentary behaviors in relation to risk of obesity and type 2 diabetes mellitus in women. JAMA 2003, 289: |785-|79|.

13. Wannamethee SG, Camargo CA Jr, Manson JE, Willett WC, Rimm $E B$ : Alcohol drinking patterns and risk of type 2 diabetes mellitus among younger women. Arch Intern Med 2003, 163:1329-1336.

14. Hu FB, van Dam RM, Liu S: Diet and risk of Type II diabetes: the role of types of fat and carbohydrate. Diabetologia 200I, 44:805-8I7.

15. Harris R, Donahue K, Rathore SS, Frame P, Woolf SH, Lohr KN: Screening adults for type 2 diabetes: a review of the evidence 
for the U.S. Preventive Services Task Force. Ann Intern Med 2003, 138:215-229.

16. Intensive blood-glucose control with sulphonylureas or insulin compared with conventional treatment and risk of complications in patients with type 2 diabetes (UKPDS 33). UK Prospective Diabetes Study (UKPDS) Group. Lancet 1998, 352:837-853.

17. Tight blood pressure control and risk of macrovascular and microvascular complications in type 2 diabetes: UKPDS 38. UK Prospective Diabetes Study Group. BMJ 1998, 317:703-7|3.

18. Colhoun HM, Betteridge DJ, Durrington PN, Hitman GA, Neil HA, Livingstone SJ, Thomason MJ, Mackness MI, Charlton-Menys V, Fuller $\mathrm{J}, \mathrm{CARDS}$ investigators: Primary prevention of cardiovascular disease with atorvastatin in type 2 diabetes in the Collaborative Atorvastatin Diabetes Study (CARDS): multicentre randomised placebo-controlled trial. Lancet 2004, 364:685-696.

19. Ezzati M, Oza S, Danaei G, Murray CJ: Trends and cardiovascular mortality effects of state-level blood pressure and uncontrolled hypertension in the United States. Circulation 2008, I 1 7:905-9|4.

20. Murray CJ, Kulkarni SC, Ezzati M: Understanding the coronary heart disease versus total cardiovascular mortality paradox: a method to enhance the comparability of cardiovascular death statistics in the United States. Circulation 2006, I | 3:207|-208I.

Publish with Bio Med Central and every scientist can read your work free of charge

"BioMed Central will be the most significant development for disseminating the results of biomedical research in our lifetime. "

Sir Paul Nurse, Cancer Research UK

Your research papers will be:

- available free of charge to the entire biomedical community

- peer reviewed and published immediately upon acceptance

- cited in PubMed and archived on PubMed Central

- yours - you keep the copyright

Submit your manuscript here:

http://www.biomedcentral.com/info/publishing_adv.asp 